\title{
SEM INVESTIGATION OF POLLEN TAXA IN HONEYS FROM AUTOCHTONE APIS CERANA IN GODAVARI, LALITPUR DISTRICT, NEPAL
}

\author{
K.N. Paudayal ${ }^{1}$ and I. Gautam ${ }^{2}$
}

\section{ABSTRACT}

Pollen analysis of 8 multifloral honey samples collected from 4 locations of Godavari, Lalitpur district, Nepal was performed using Scanning Electron Microscope (SEM). In this investigation, a wide range of foraging plant sources for Apis cerana honey bees was identified which demonstrates the adequate potential for expanding and sustaining beekeeping in this area. The palynological assemblage of a total of 44 species of pollen flora representing 28 families was identified to the generic and some up to species level. Some of the pollen grains identified to only families, belong to Acanthaceae, Apiaceae, Araliaceae, Chenopodiaceae, Compositae, Lamiaceae, Loranthaceae, Meliaceae, Poaceae, Rosaceae, Rutaceae and Pteridaceae. The pollen assemblages in honeys were mostly belonging to angiosperms while the gymnosperm pollen was completely absent. One pteridophyte spore belonging to family Pteridaceae recovered. In this paper the morphology of the pollen grains based on SEM observation are described and the importance of the systematic documentation of various bee flora are discussed.

Key words: pollen analysis, multifloral honey, autochtone honey bee, SEM, Kathmandu Valley

\section{INTRODUCTION}

Honey is natural complex food product produced by bees from nectar of plants and also from honeydew. Honey contains a significant quantity of pollen which is the only proteic food within the bee hive and used for feeding the larvae and the young bees. The combination of wind and insect-pollinated taxa found in a honey sample often produce a pollen spectrum that is a valuable tool for identifying the geographical origin of the honeys for the presence of a combination of pollen that is typical only to that particular location (Louveaux et al. 1978) and to identify the genera of the plants the honeys bees visited. In Nepal, bee-keeping is practiced in many areas, characterized by a remarkable richness of bee floras. Information gained from a given honey sample is useful when substantiating claims of a particular honey source and is also of great importance for quality control and helps to ascertain whether honey is adulterated or not (Maurizio 1951, Molan 1998, Louveaux et al. 1978, Terrab et al. 2003). The element content of bees and bee products recognized as useful indicator of the presence of specific minerals within their forage area (Crane 1984). Honey may also contain enzymes of plant origin from nectar or honeydew, and possibly from pollen (Crane 1981). Therefore, a pollen analysis of honey not only gives the palynological composition itself but also provides a broad knowledge of floral assemblage of an area where the bees are foddering.

Light microscopy (LM) in melissopalynology is used to identify and interpret the pollen spectrum of a particular sample. Whereas several workers used Scanning Electron Microscopy (SEM) for morphological comparisons and taxonomy (Skvarla and Larson 1965, Ridgeway and Skvarla 
1969), to create new terminology for describing pollen ornamentation (Rowley et al. 1988, Vezey et al. 1991), developing a numerical approach to pollen sculpturing (Vezey et al. 1991), and even computer analysis of the exine (Vezey and Skvarla 1990). There are several reports available on pollen morphology from various parts of the world such as SEM micrographs and some atlases (Ogden et al. 1974, Moore et al. 1991, Nilsson et al. 1977, Bassett et al. 1978, Moar, 1993, Qiao 2004) and few SEM micrographs for the pollen presentation (Adams and Morton 1972, 1974, 1976, 1979, Bambara and Leidy 1991, Jones et al. 1995, Wei et al. 2003); however, the majority focuses only on LM (Herrera and Urrego 1996, Beug 2004). Most entomopalynological studies use LM for pollen analyses (Jones and Coppedge 1998). However, SEM is useful in melissopalynology (Van Laere et al. 1969) with its use in honey study.

The earliest research on the pollen analysis of honey dates back to 1895 by Pfister with examination of pollen contents of various Swiss, French, and other European honeys. He was able to identify many of the pollen grains he found because of earlier studies of pollen morphology, structure, and identification of European pollen types by botanists including Guillemin in 1825, Fritsche in 1832, Mohl in 1834, and Fischer in 1890 (Woodehouse 1935). Further, there were several works carried out on melissopalynology (Fehlman 1911, Armbruster 1929, 1934-35, Griebel 1931, Fossel 2000, Köppler et al. 2007) including the most authoritative five-volume work of Zander (1935, 1937, 1941, 1949, 1951), which laid the foundation for melissopalynology research in Europe. The first melissopalynological investigations in the US honey was carried out by Young (1908) and Swiss honey by FehIman (1911) (Maurizio 1951). Works of Betts $(1923,1925)$ and Allen (1928) on English honey, made other research advancements in melissopalynology. Todd and Vansell in the US (1942) investigated the relationship and importance of pollen in honey. Chen and Shen (1990) used SEM to examine the pollen of Formosan honey. The first melissopalynological study in Africa is reported by Smith in 1956 (Sowunmi 1976). In India, Sen and Banerjee (1956) analyzed the pollen content of honey samples obtained from a garden near Calcutta and observed an over abundance of anemophilous pollen grains. Vishnu-Mittre (1958) examined the pollen contents of ten samples of Indian honey. Other melissopalynological works from different parts of India were also carried out (Sen and Banerjee 1956, Vishnu-Mittre 1958, Nair 1964, Sharma and Nair 1965, Suryanarayana and Thakar 1966, Suryanarayana et al. 1977, Chanda and Ganguly 1981, Chaturvedi 1983, 1989). In addition, the melissopalynological studies to identify bee plants in different parts of the world (Varis et al. 1982, Feller-Demalsy 1983, Agwu et al. 1985, Moar 1985, Feller-Demalsy et al. 1987, Tellaria 1988, Chen and Shen 1990, Jato et al. 1991, 1994, Blackburn and Ford 1993, Valencia-Barrera et al. 1994, Loublier et al. 1994, Lobreau-Callen and Damblon 1994) including Hindu Kush-Himalaya region (Focke 1968, Deh-Feng and Wen Cheng 1981, Yue-Zhen 1984, Saraf 1972, Atwal and Goyal 1974, Singh 1983, Singh 1989, ICIMOD 1996) revealed the potential resources for apiculture industry.

There is some information available on the honey plant resources in Nepal. The sources of informations on bee plants in Nepal are field surveys and melissopalynology (Partap 1997). The bee flora of Nepal have been previously surveyed by Kafle $(1984,1992)$, Maskey (1989, 1992), Partap and Verma (1996). A list of 156 (Kafle 1984) and 113 (Partap and Verma 1996) bee flora of Kathmandu valley was recorded. Partap (1997) has also reported some bee 
plants from Nepal. Diverse bee floras reported from different agro-ecological zones of Nepal represent various agricultural, horticultural, and forage crops, ornamental plants, avenue trees, wild plants, and forest trees (Partap 1997). Further, SEM study of pollen morphology of the Himalayan Viola had been carried out by Shrestha et al. (2005). In spite of elucidation of the melissopalynological advancement in the world very few works have been carried out in Nepal. Nevertheless, the melissopalynological studies of $A$. cerana honey from Jumla, Nepal recorded the occurrence of about 103 plant species constituting the bee forage sources of that region (ICIMOD 1996). The detail investigation on pollen spectrum of Nepali honeys carried out by Joshi (1999). He identified total 51 pollen types in Apis dorsata, A. cerana and A. mellifera honeys from Chitwan district, 50 pollen types in $A$. cerana and $A$. mellifera honeys from Kathmandu valley, 16 pollen types in $A$. cerana honeys from Jajarkot district, 49 pollen types in $A$. cerana honeys from Dadeldhura district, 43 pollen types in $A$. cerana honeys from Jumla district, and 25 pollen types in $A$. cerana honeys from Langtang. His study represents the major findings in melissopalynology in Nepal indicating the ecological origins of the honeys produced by Apis dorsata, A. cerana and $A$. mellifera and provided important contributions to the knowledge of the wild and cultivated bee forage. In addition, over nineteen species of pollen flora belonging to thirteen families were recovered from four pollen load samples from honey bee $A$. cerana collected in Jajarkot district, mid-western region, Nepal using light microscopy along with scanning electron microscopy for the first by Paudayal and Gautam (2011a) and eight pollen types representing seven families of foraging plant sources for the same autochtone species of honey bee in Bajhang district, west Nepal (Paudayal and Gautam 2011b). The previous melissopalynological investigations conducted on Nepali honeys limited to light microscopy (LM) to identify and interpret the pollen spectrum of a particular honey sample. Therefore, the present investigation deals with the SEM description of the pollens of autochtone honey bee A. cerana honeys collected from Godavari area, southern part of the Kathmandu Valley, Nepal. The morphological characters of the pollen of 44 species belonging to 28 families described in this paper are certainly not possible with a single LM study.

\section{STUDY AREA}

Godavari, in Lalitpur district, in the Bagmati Zone of central Nepal, with a latitude of 27.6 (27 $\left.36^{\prime} 0 \mathrm{~N}\right)$ and a longitude of $85.4\left(85^{\circ} 24^{\prime} 0 \mathrm{E}\right)$, is situated around at an altitude of $1,1455 \mathrm{~m}$ to 2,765 $\mathrm{m}$ above the mean sea level. Godavari lies at the foot of Phulchowki mountain, offers rich flora and fauna and provides access to Nepal's famous Botanical garden which has a large collection of plants. A marble quarry that has operated in the region has caused mass deforestation, exposing the Phulchowki mountain.

\section{MATERIALS AND METHODS}

The bee hives examined were either those of exotic $A$. mellifera or autochtone $A$. cerana bee colonies. The honey samples produced by $A$. cerana were obtained from sealed honey combs from four randomly selected locations of Godavari area. The study comprised the analysis of 8 honey samples. The honey samples from $A$. cerana were taken to the palynology section of the Institute of Palaeontology, Vienna University, Austria in sealed plastic jars for Scanning Electron Microscope (SEM) observation. The samples were diluted with distilled water and 
centrifuged at $3000 \mathrm{rpm}$ (rotation per minute) for 3 minutes to collect the pollen. This process was repeated for several times to collect a significant amount of pollen. The samples were washed with glacial acetic acid two times and then proceeded for acetolysis to remove the cellulose and cell content in the pollen grains. This included the treatment with a solution containing acetic anhydride $\left(\mathrm{CH}_{3} \mathrm{CO}\right)_{2} \mathrm{O}$ and concentrated sulphuric acid $\left(\mathrm{H}_{2} \mathrm{SO}_{4}\right)$ in a ratio of 9:1 (Erdtman 1954). The samples were then kept in water bath for 5 minutes and washed with glacial acetic acid and water respectively. At the end, samples were kept in glycerin and proceeded for Light Microscopic (LM) study.

After examining the pollen grain under LM and taking LM photographs the same pollen grain was brought to the edge of the glycerin on the glass slide using the specially adapted needle with a human hair glued at the tip (Zetter 1989, Ferguson et al. 2007). With the help of needle the pollen was transferred to a SEM stub to which a drop of absolute ethanol $\left(\mathrm{C}_{2} \mathrm{H}_{5} \mathrm{OH}\right)$ was applied with a pipette simultaneously. The SEM stub was kept under a binocular microscope at the required magnification. Care was taken while dropping the absolute ethanol ( $99 \%$ pure), not to wash the pollen grain off the stub. The pollen grains were then coated with gold in a BIORAD Sputter Coater for four minutes. It was then followed by the examination of the pollen with Jeol JSM 6400 Scanning Electron Microscope at $10 \mathrm{kV}$ at different magnification and orientation. SEM photographs were taken with the camera attached to the microscope using AGFA APX 100 (100 ASA) black and white film. This was followed by development of the film and photographs in the dark room.

Pollen grains were generally identified according to their physical appearance. The criteria of identification used according to the position and number of apertures, the shape and size of the pollen grain as a whole, and the elaborate, fine structure (ornamentation) on the sexine; the sculptured exine. There are mainly three types of apertures; porate, having isodiametric pores and colpate, having apertures that are long, boat shaped with pointed ends. Sometimes the pore and colpus in a pollen grains combined together to form colporate apertures. If arranged equidistantly round the equator of the pollen grain; they are assigned the prefix; zono-. If scattered all over, the prefix panto-. The number of aperture is also indicated by prefixes; mono- for one aperture, di-for two apertures, tri- for three apertures, tetra for four and penta or poly- for numerous apertures. Morphological classes of pollen grains on the basis of aperture and symmetry can be viewed from equatorial as well as polar axis. Each pollen grain varies in their ultra-structures in the species level which helps for accurate taxonomical groupings (Erdtman et al. 1952). The SEM study is very helpful to identify the pollen to lower taxonomical level as the details in the tectum can be measured in microns in high magnification (Ferguson et al. 2007). 

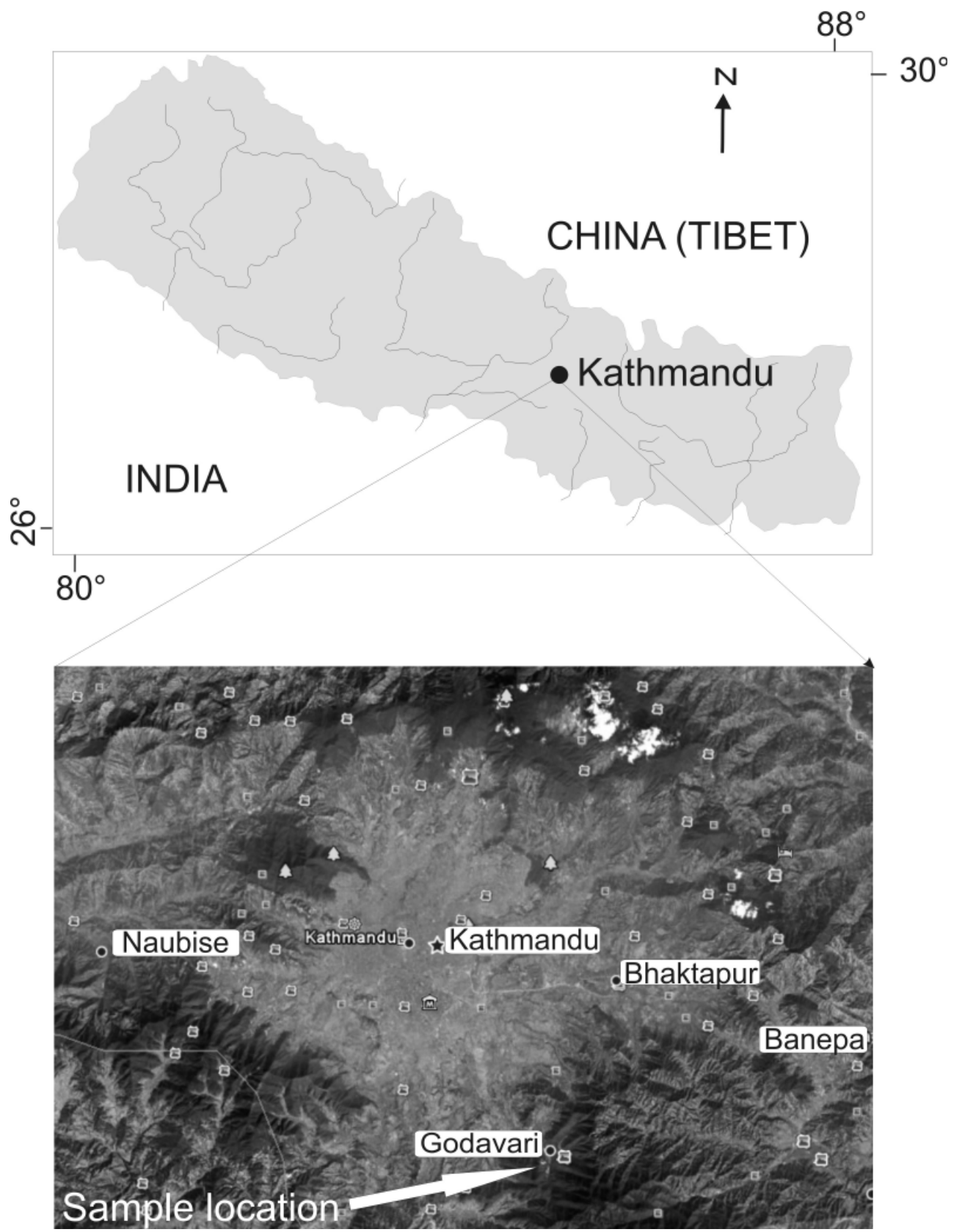

Fig. 1. Location of the sample area indicated by arrow. 


\section{RESULTS AND DISCUSSION}

The melissopalynological investigation of 8 A. cerana honey samples from Godavari area revealed 44 species of plant taxa belonging to 28 families (Table 1). The palynological assemblages identified to the generic and some up to species level belong to Aesandra sp., Alnus sp., Artemisia sp., Brassica sp., Cornus sp., Corylus sp., Fagopyrum sp., Fraxinus sp., Grevillea sp., Impatiens sp., Jasminum sp., Justicia sp., Ligustrum sp., Melia azederach, Myrica esculenta, Polygonum sp., Quercus lanata, Rhododendron sp., Salix sp., Sarcococca sp., Syzygium sp., and Urtica sp. etc. Some of the pollen grains identified to only families, belong to, Acanthaceae, Apiaceae, Araliaceae, Chenopodiaceae, Compositae, Lamiaceae, Loranthaceae, Meliaceae, Poaceae, Rosaceae, Rutaceae and Pteridaceae. The pollen assemblages were mostly belonging to angiosperms while the gymnosperm pollen was completely absent. One pteridophyte spore belonging to family Pteridaceae recovered from the sample.

Table 1. Floral diversity of the pollen assemblage in a multifloral honey from Godavari area.

\begin{tabular}{|l|l|l|l|}
\hline Family & $\begin{array}{l}\text { N u m b e r o f } \\
\text { genera }\end{array}$ & Family & $\begin{array}{l}\text { N u m b e r o f } \\
\text { genera }\end{array}$ \\
\hline Acanthaceae & 4 & Loranthaceae & 1 \\
\hline Apiaceae & 1 & Meliaceae & 2 \\
\hline Araliaceae & 2 & Myricaceae & 1 \\
\hline Balsaminaceae & 1 & Myrtaceae & 1 \\
\hline Betulaceae & 1 & Oleaceae & 4 \\
\hline Brassicaceae & 1 & Poaceae & 1 \\
\hline Buxaceae & 1 & Polygonaceae & 2 \\
\hline Chenopodiaceae & 1 & Proteaceae & 1 \\
\hline Compositae & 5 & Rosaceae & 4 \\
\hline Cornaceae & 1 & Rutaceae & 1 \\
\hline Corylaceae & 1 & Salicaceae & 1 \\
\hline Ericaceae & 1 & Sapotaceae & 1 \\
\hline Fagaceae & 1 & Urticaceae & 1 \\
\hline Lamiaceae & 1 & Pteridaceae & 1 \\
\hline
\end{tabular}




\section{A. ANGIOSPERMS}

\section{Family Acanthaceae}

Acanthaceae gen. et spec. indet. (1)

Plate I, Figs. 1-2

Shape: Prolate.

Size: Polar axis $45 \mu \mathrm{m}$, equatorial axis $22 \mu \mathrm{m}$.

Aperture: Colporate.

Exine: $3 \mu \mathrm{m}$, sexine much thicker than nexine, sexine reticulate, the reticulations are smaller in mesoclpium while wider near the aperture area.

\section{Justicia sp.}

Plate I, Figs. 3-4

Shape: Prolate.

Size: Polar axis $40 \mu \mathrm{m}$, equatorial axis $28 \mu \mathrm{m}$.

Aperture: Colporate.

Exine: $1.5 \mu \mathrm{m}$, sexine as thick as nexine, sexine reticulate, lumina microreticulate, sexine around aperture rugulate, perforate and granulate.

Acanthaceae gen. et spec. indet. (2)

Plate I, Figs. 5-6

Shape: Prolate.

Size: Polar axis $27 \mu \mathrm{m}$, equatorial axis $15 \mu \mathrm{m}$.

Aperture: Tricolporate.

Exine: $1.5 \mu \mathrm{m}$, sexine as thick as nexine, sexine reticulate, rugulate, granulate and perforate near aperture, lumina wider near the aperture area compared to mesocolpium. Aperture wide and disctinct.

Acanthaceae gen. et spec. indet. (3)

Plate I, Figs. 7-8

Shape: Prolate.

Size: Polar axis $25 \mu \mathrm{m}$, equatorial axis $13 \mu \mathrm{m}$.

Aperture: Colporate.

Exine: $1.5 \mu \mathrm{m}$, sexine as thick as nexine, sexine reticulate, lumina microreticulate, area around the aperture perforate and granulate, muri sometimes granulate. 


\section{PLATE-I}
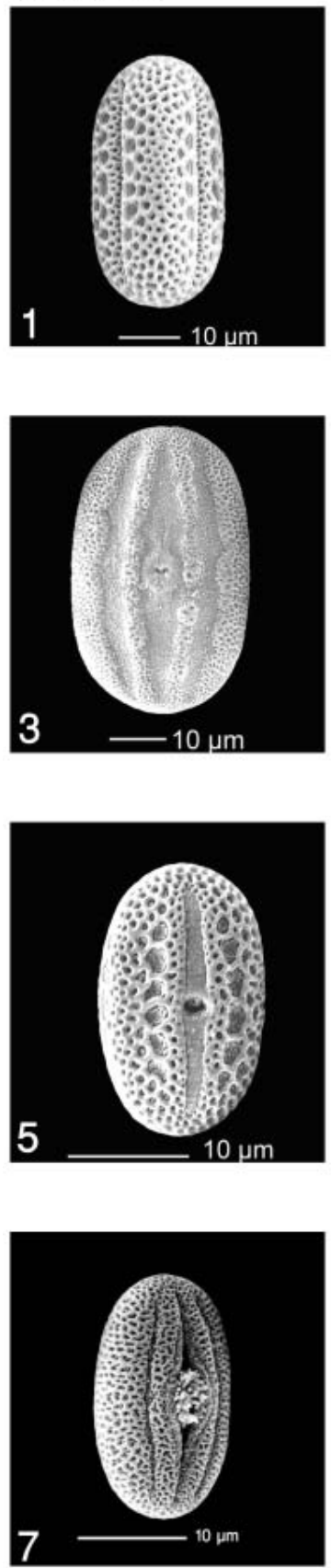
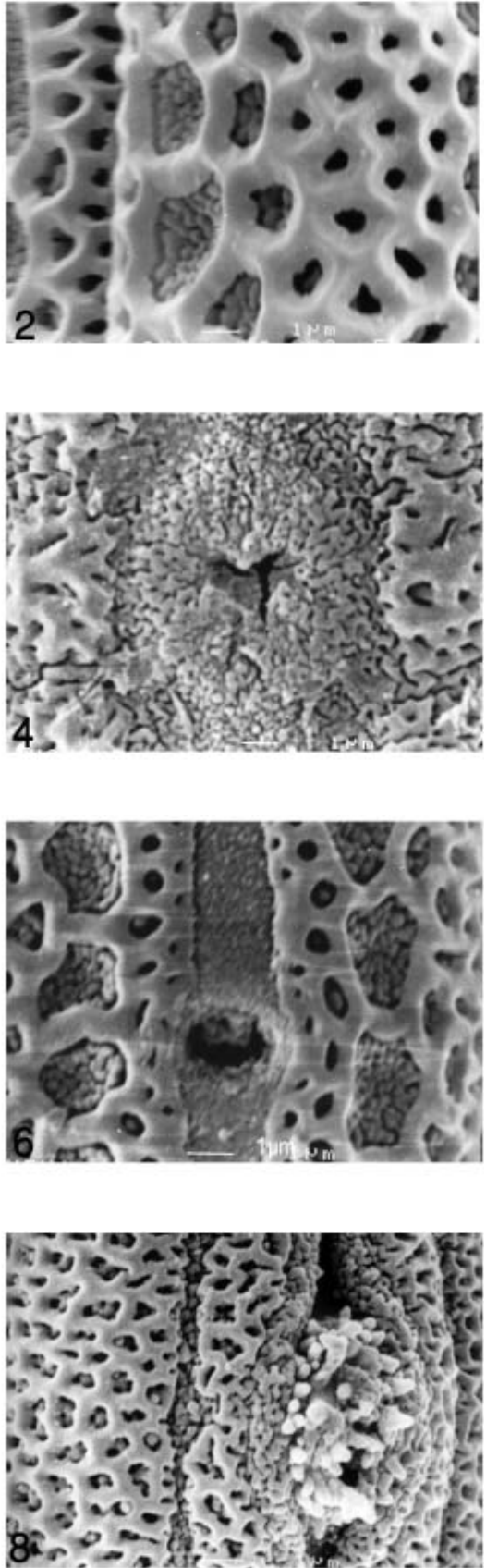


\section{Family Apiaceae}

Apiaceae gen. et spec. indet.

Plate II, Figs. 9-10

Shape: Prolate.

Size: Polar axis $26 \mu \mathrm{m}$, equatorial axis $12 \mu \mathrm{m}$.

Aperture: Tricolporate, endoaperture lolongate.

Exine: $1 \mu \mathrm{m}$, sexine shortly striate, striae up to $7 \mu \mathrm{m}$ long and sometimes crisscrossed, granulate.

\section{Family Araliaceae}

Araliaceae gen. et spec. indet. (1)

Plate II, Figs. 11-12

Shape: Prolate, triangular in polar view.

Size: Equatorial axis $18 \mu \mathrm{m}$.

Aperture: Tricolporate, endopore is lolongate and distinct.

Exine: $1.5-2 \mu \mathrm{m}$, tectate, tectum micro-reticulate to reticulate, lumina heterobrochate, broader in mesocolpium than in aperture areas, sexine as thick as nexine but slightly thicker in the polar area.

Araliaceae gen. et spec. indet. (2)

Plate II, Figs. 13-14

Shape: Prolate.

Size: Polar axis $18 \mu \mathrm{m}$.

Aperture: Tricolporate

Exine: $1.5-2 \mu \mathrm{m}$, tectate, tectum micro-reticulate to reticulate, lumina heterobrochate, broader in mesocolpium than in aperture areas. Colpi nearly as long as polar axis.

\section{Family Balsaminaceae}

\section{Impatiens $\mathrm{sp}$.}

Plate II, Figs. 15-16

Shape: Oblate, rectangular in polar view.

Size: Equatorial axis $33 \mu \mathrm{m}$.

Aperture: Tetracolpate, colpi short.

Exine: $1.5 \mu \mathrm{m}$, sexine as thick as nexine, reticulate, rudimentary columellae are present in lumina. 

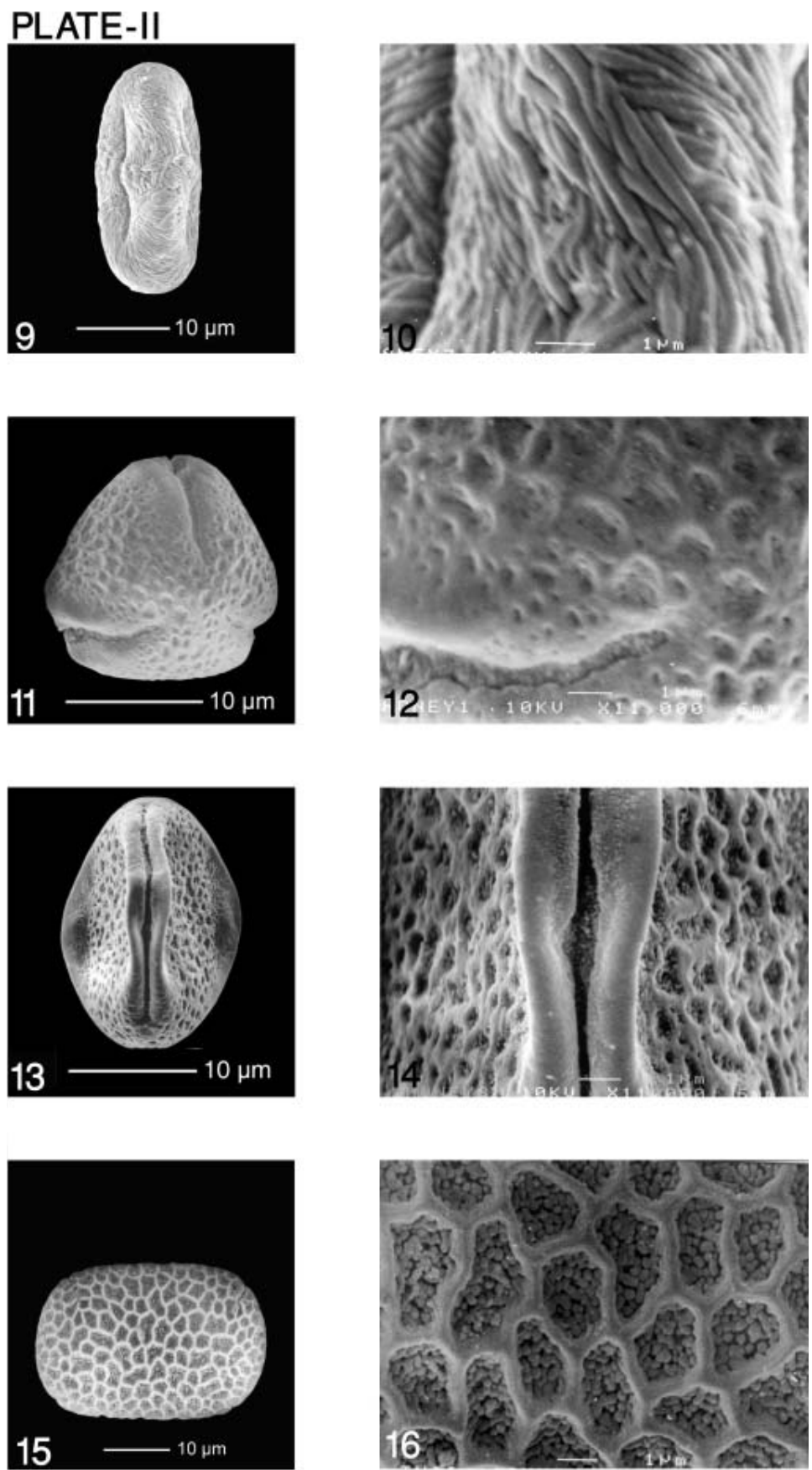


\section{Family Betulaceae}

Alnus sp.

Plate III, Figs. 17-18

Shape: Oblate, penta-angular in polar view.

Size: $15-20 \mu \mathrm{m}$

Aperture: Pentaporate, pori vestibulum type, neighbouring pori connected by archs or bands of nexinous thickening.

Exine: $1.5 \mu \mathrm{m}$, tectum consists of irregular rugulae with very small spinules (microechinate).

Sexine slightly thicker than nexine.

\section{Family Brassicaceae}

\section{Brassica sp.}

Plate III, Figs. 19-20

Shape: Slightly prolate, circular in polar view.

Aperture: Tricolpate

Exine: $1.3 \mu \mathrm{m}$, tectum uniformaly reticulate, the colpi area is granulate.

\section{Family Buxaceae}

Sarcococca sp.

Plate III, Figs. 21-22

Shape: Spheroidal.

Size: $24-25 \mu \mathrm{m}$.

Aperture: Pantoporate, pori small and devoid of annulus.

Exine: $2 \mu \mathrm{m}$, sexine much thicker than nexine, tectum reticulate (retipilate), muri formed by rows of pila.

\section{Family Chenopodiaceae}

Chenopodiaceae gen. et spec. indet.

Plate III, Figs. 23-24

Shape: Spheroidal.

Size: 18 - $27 \mu \mathrm{m}$.

Aperture: Pantoporate, pore circular.

Exine: $1 \mu \mathrm{m}$, sexine thicker than nexine, sexine spinulate, perforate, mesoporium raised above pori, pore membrane spinulate, granulate. 
PLATE-III
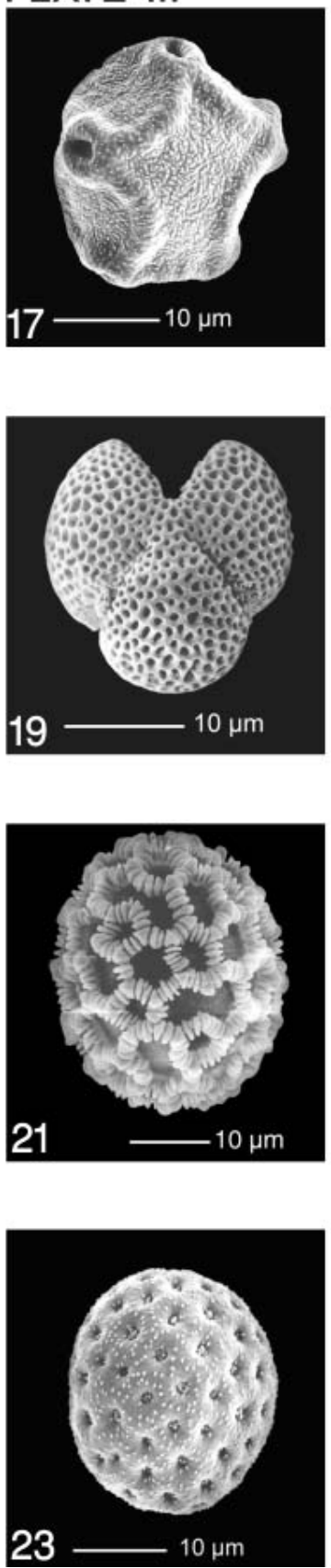
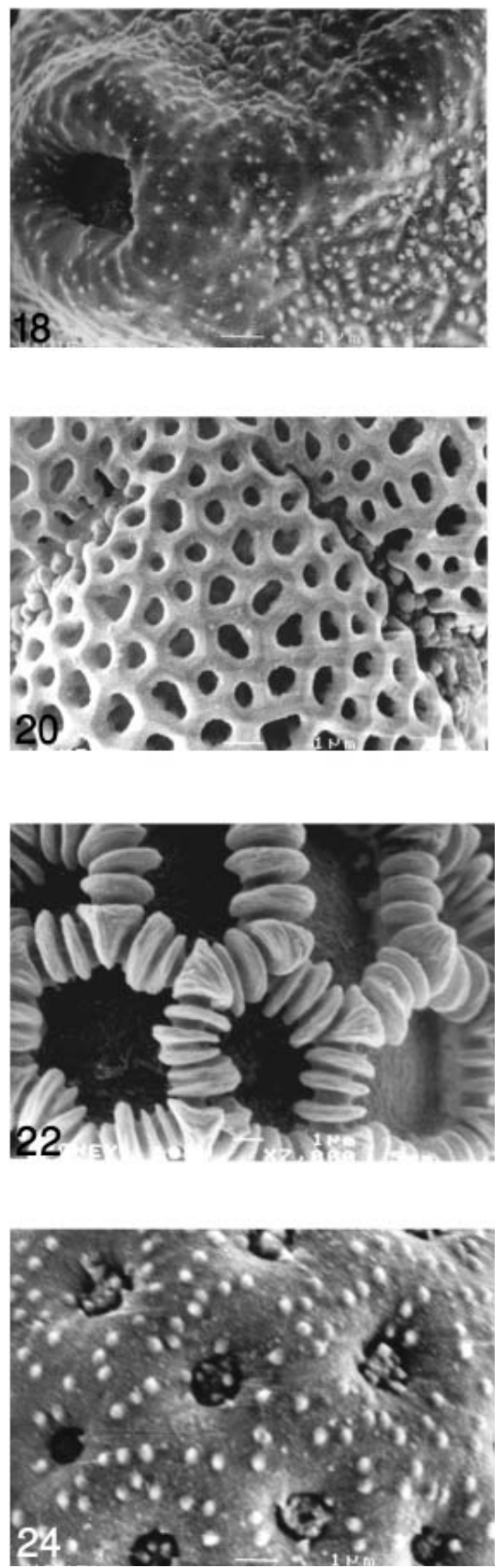


\section{Family Compositae (Asteraceae)}

\section{Artemisia sp.}

Plate IV, Figs. 25-26

Shape: Prolate, circular in polar view, lobate.

Size: Polar axis $20 \mu \mathrm{m}$.

Aperture: Tricolporate, colpi nearly as long as polar axis.

Exine: $3 \mu \mathrm{m}$, sexine much thicker than nexine, distinctly stratified, sexine in mesocolpium thicker than in the colpi area forming a margo, sexine microechinate and granulate, granules uniformly distributed between the spinules.

Tubiflorae Compositae gen. et spec. indet. (1)

Plate IV, Figs. 27-28

Shape: Prolate, circular in equatorial view.

Size: Polar axis $29 \mu \mathrm{m}$, equatorial axis $26 \mu \mathrm{m}$.

Aperture: Tricolporate, colpi long.

Exine: $2 \mu \mathrm{m}$ (without spines), sexine as thick as nexine, spiny, perforate, spines $>7 \mu \mathrm{m}$ in length, with a broad perforated base and long apices.

Tubiflorae Compositae gen. et spec. indet. (2)

Plate IV, Figs. 29-30

Shape: Prolate, in equatorial view elliptical, in polar view circular, lobate.

Size: Polar axis $24 \mu \mathrm{m}$, equatorial axis $20 \mu \mathrm{m}$.

Aperture: Tricolporate.

Exine: $4 \mu \mathrm{m}$, sexine very thick, micro perforate, granulate, spiny, lumina of the perforations larger in size at the base of the spines.

Tubiflorae Compositae gen. et spec. indet. (3)

Plate IV, Figs. 31-32

Shape: Spheroidal to oblate, in polar view circular, lobate.

Size: Equatorial axis $21 \mu \mathrm{m}$.

Aperture: Tricolporate.

Exine: $6 \mu \mathrm{m}$, sexine much thicker in mesocolpium than in the colpi area, perforate, spiny and occasionally granulate, spines with broad base, short (1-2 $\mu \mathrm{m})$ and extremely perforated. 

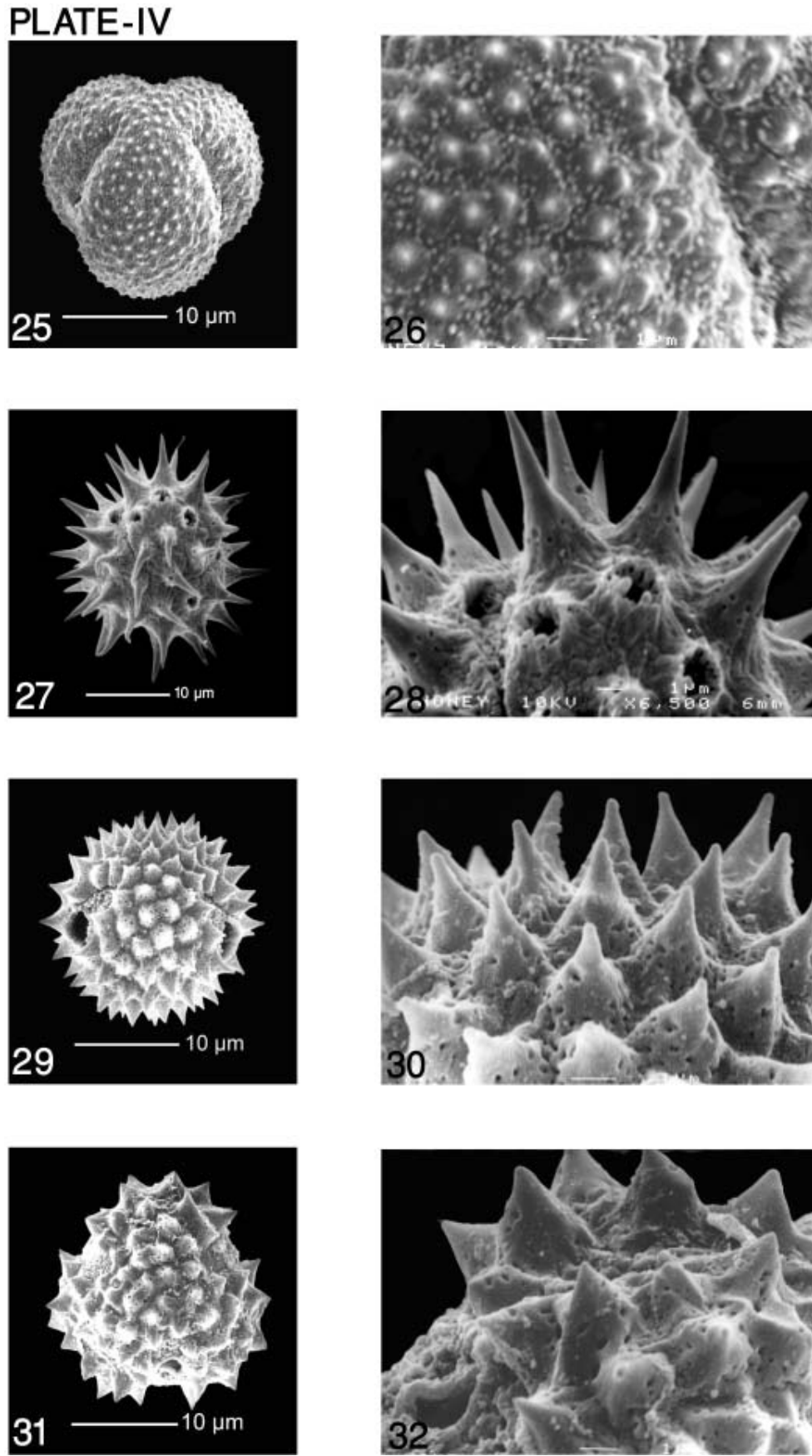
Tubiflorae Compositae gen. et spec. indet. (4)

Plate V, Figs. 33-34

Shape: Prolate to spheroidal, lobate.

Size: $19 \mu \mathrm{m}$.

Aperture: Tricolporate.

Exine: $1 \mu \mathrm{m}$, sexine as thick as nexine, spiny and granulate, tectum and spines with only very few perforations.

\section{Family Cornaceae}

\section{Cornus sp.}

Plate V, Figs. 35-36

Shape: Prolate.

Size: Polar axis $20 \mu \mathrm{m}$.

Aperture: Tricolporate

Exine: $1.5-2 \mu \mathrm{m}$, tectate, tectum micro-echinate, Colpi nearly as long as polar axis.

\section{Family Corylaceae}

\section{Corylus sp.}

Plate V, Figs. 37-38

Shape: Oblate, triangular in polar view.

Size: $26-28 \mu \mathrm{m}$.

Aperture: Triporate.

Exine: $1.5 \mu \mathrm{m}$, sexine is slightly thicker than nexine, nexine is poorly developed near pori, surface sculpture scabrate and slightly regulate and microechinate.

\section{Family Ericaceae}

\section{Rhododendron sp.}

Plate V, Figs. 39-40

Shape: Tetrahedral tetrad.

Size: $26-30 \mu \mathrm{m}$.

Aperture: Monads tricolporate.

Exine: $1.5 \mu \mathrm{m}$, sexine as thick as nexine, sexine rugulate in mesocolpium areas and perforate to foveolate in distal pole areas, irregularly distributed granules. 
PLATE-V
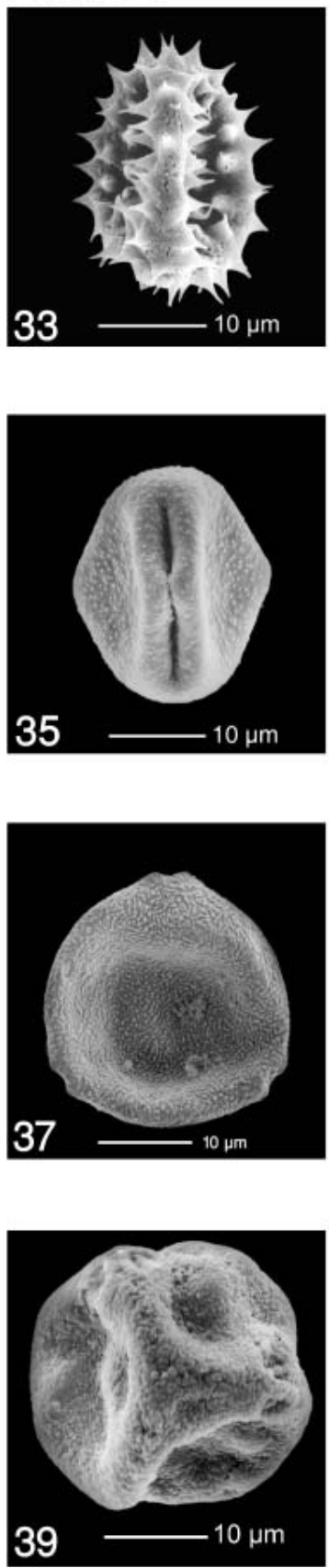
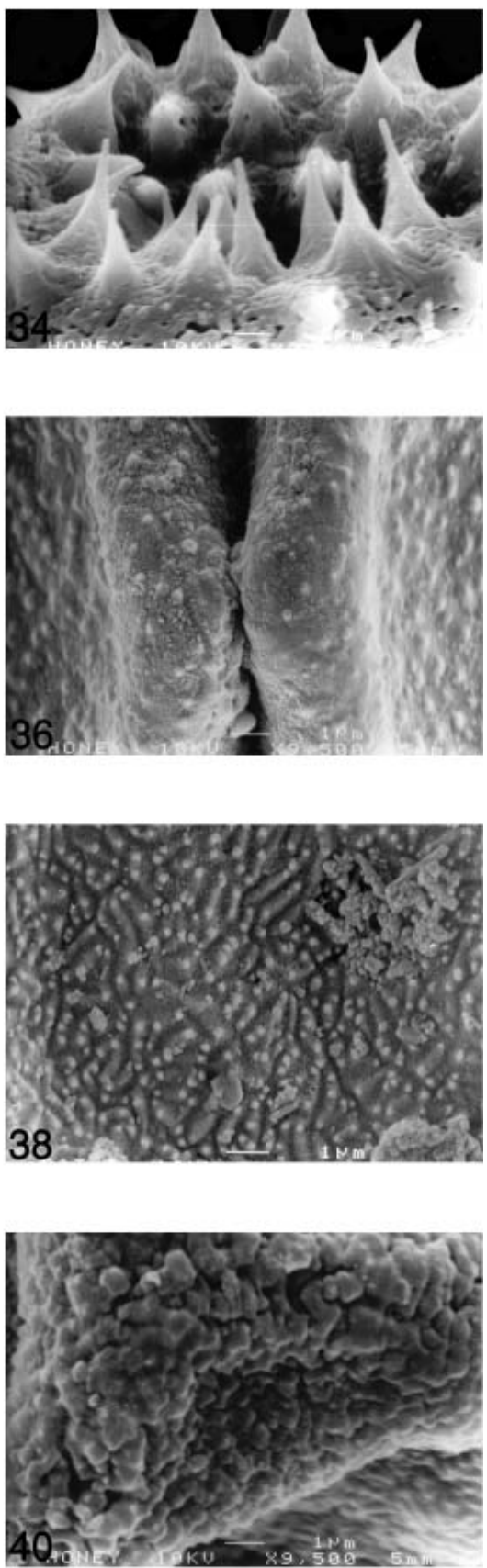


\section{Family Fagaceae}

\section{Quercus lanata Smith}

Plate VI, Figs. 41-42

Shape: Prolate.

Size: Polar axis $22 \mu \mathrm{m}$, equatorial axis $16 \mu \mathrm{m}$.

Aperture: Tricolporate.

Exine: $1-1.3 \mu \mathrm{m}$, tectum consists of small $(<1 \mu \mathrm{m})$ randomly oriented, sometimes bifurcated rods, which are not uniformly distributed. The rods sometimes accumulated to make clusters.

\section{Family Labiatae (Lamiaceae)}

Labiatae gen. et spec. indet.

Plate VI, Figs. 43-44

Shape: Oblate, semicircular in polar view.

Size: Equatorial axis $38-42 \mu \mathrm{m}$.

Aperture: Hexacolpate, colpi long and 6-7 $\mu$ m wide.

Exine: $1.5 \mu \mathrm{m}$, sexine slightly thicker than nexine, sexine suprareticulate, muri less than $1 \mu \mathrm{m}$, lumina large up to $2 \mu \mathrm{m}$.

\section{Family Loranthaceae}

Loranthaceae gen. indet.

Plate VI, Figs. 45-46

Shape: Oblate, amb angular and lobate.

Size: Equatorial $30 \mu \mathrm{m}$.

Aperture: Tricolporate (syncolporate).

Exine: $1-2 \mu \mathrm{m}$, sexine finely granulate.

\section{Family Meliaceae}

\section{Melia azederach}

Plate VI, Figs. 47-48

Shape: Prolate

Size: Polar $40 \mu \mathrm{m}$, Equatorial $30 \mu \mathrm{m}$

Aperture: Tetracolporate

Exine: 1-2 $\mu \mathrm{m}$, sexine regulate and granulate. 

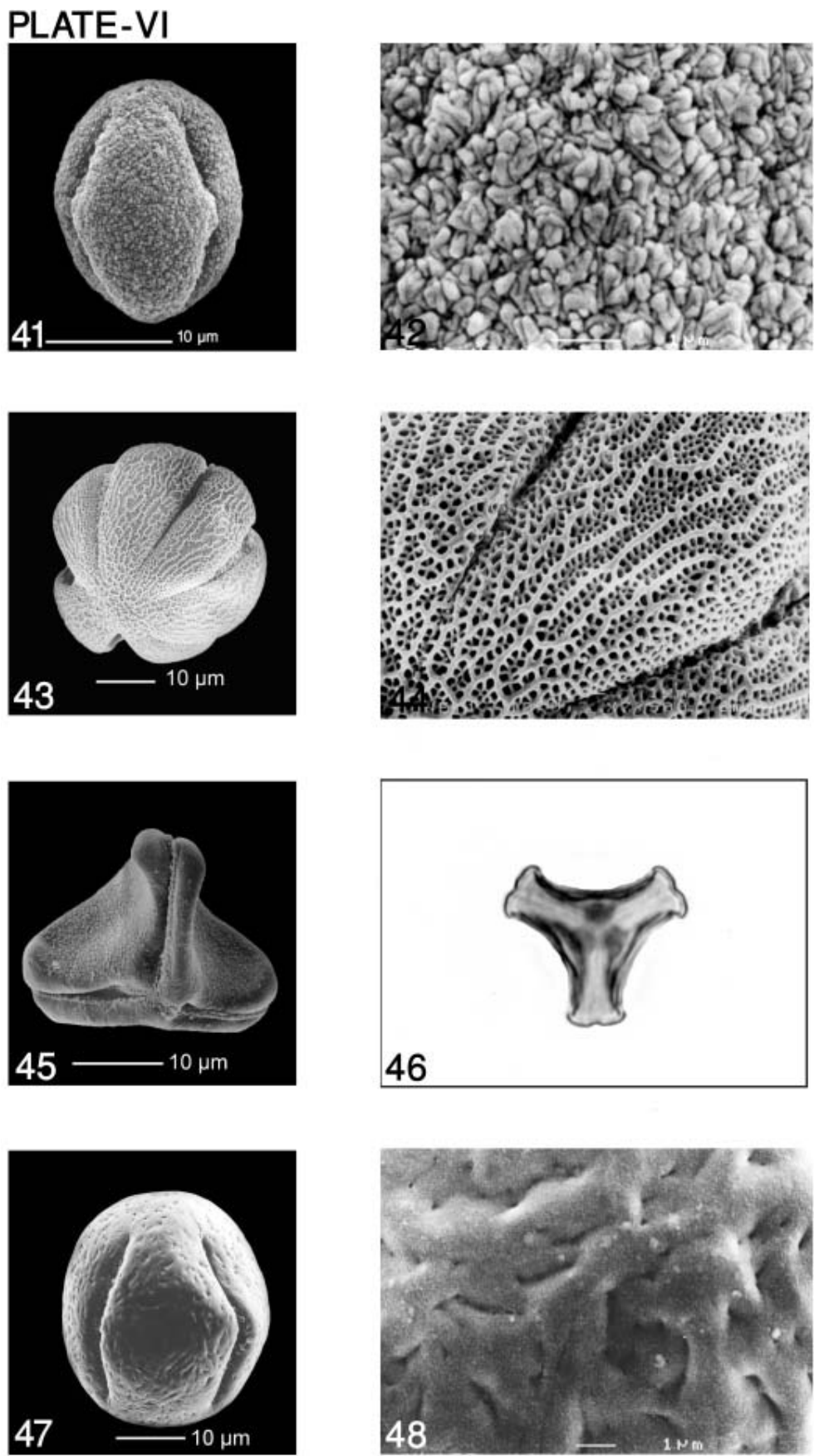
Meliaceae gen. et spec. indet

Plate VII, Figs. 49-50

Shape: Prolate

Size: Polar $22 \mu \mathrm{m}$

Aperture: Tetracolporate

Exine: 1-2 $\mu \mathrm{m}$, sexine regulate and finely granulate.

\section{Family Myricaceae}

Myrica esculenta Buch.-Ham. ex D. Don

Plate VII, Figs. 51-52

Shape: Oblate, triangular in polar view.

Size: $19 \mu \mathrm{m}$.

Aperture: Triporate.

Exine: $1 \mu \mathrm{m}$, sexine is thicker than nexine near and around pori, sexine regularly microechinate.

\section{Family Myrtaceae}

\section{Syzygium sp.}

Plate VII, Figs. 53-54

Shape: Oblate, triangular and lobed in polar view.

Size: $20-21 \mu \mathrm{m}$.

Aperture: Trisyncolporate.

Exine: $1 \mu \mathrm{m}$, sexine as thick as nexine, tectum is coarsely rugulate, perforate.

\section{Family Oleaceae}

\section{Fraxinus sp. (1)}

Plate VII, Figs. 55-56

Shape: Prolate, in equatorial view circular and lobate.

Size: $25 \mu \mathrm{m}$.

Aperture: Tricolporate.

Exine: $1 \mu \mathrm{m}$, sexine is thicker than nexine, reticulate, lumina homobrochate and polygonal in shape, muri are distinctly granulate. 

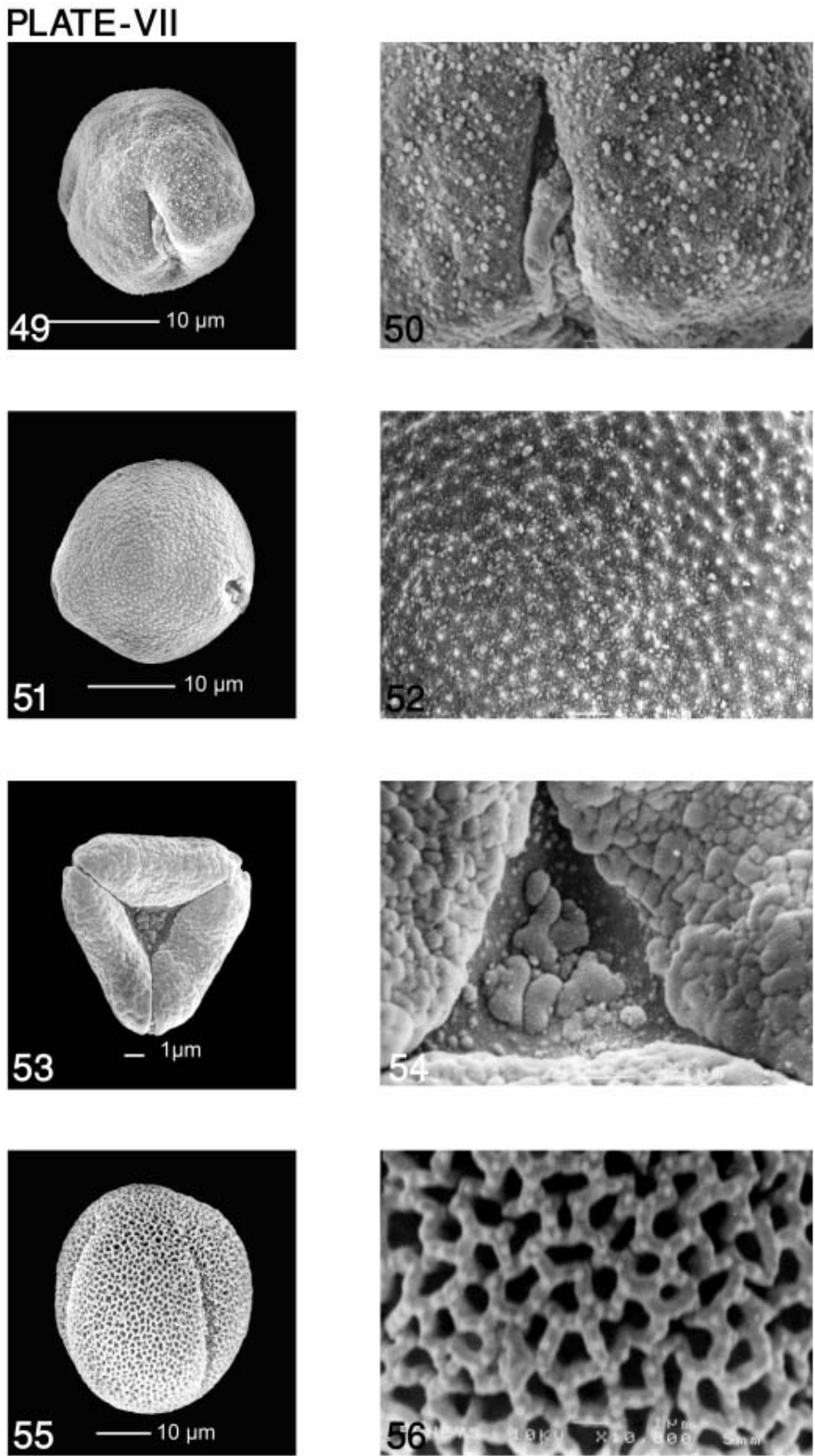
Fraxinus sp. (2)

Plate VIII, Figs. 57-58

Shape: Prolate, circular in polar view.

Size: Polar axis $16 \mu \mathrm{m}$, equatorial axis $13 \mu \mathrm{m}$.

Aperture: Tricolporate, colpi are long, small circular endoapertures.

Exine: $1 \mu \mathrm{m}$, sexine thicker than nexine, tectum reticulate, lumina are heterobrochate, triangular to polygonal in shape.

\section{Jasminum sp.}

Plate VIII, Figs. 59-60

Shape: Prolate, circular in polar view.

Size: Polar axis $40 \mu \mathrm{m}$, equatorial axis $33 \mu \mathrm{m}$.

Aperture: Tricolporate, colpi are long, small circular endoapertures.

Exine: $1 \mu \mathrm{m}$, sexine thicker than nexine, tectum reticulate, lumina are heterobrochate, triangular to polygonal in shape

\section{Ligustrum $\mathrm{sp}$.}

Plate VIII, Figs. 61-62

Shape:Sub prolate.

Size: Polar axis $37 \mu \mathrm{m}$, equatorial axis $29 \mu \mathrm{m}$.

Aperture: Tricolporate.

Exine: $1.2 \mu \mathrm{m}$, sexine is thicker than nexine, reticulate, heterobrochate, muri are smooth, lumina with rudimentary columellae.

\section{Family Poaceae}

Poaceae gen. indet.

Plate VIII, Figs. 63-64

Shape: Spheroidal.

Size: $35 \mu \mathrm{m}$.

Aperture: Ulcerate, pore annulate, diameter of the pore 2-3 $\mu \mathrm{m}$.

Exine: $1 \mu \mathrm{m}$, sexine as thick as nexine, with small spiny elements throughout the sexine. 


\section{PLATE-VIII}
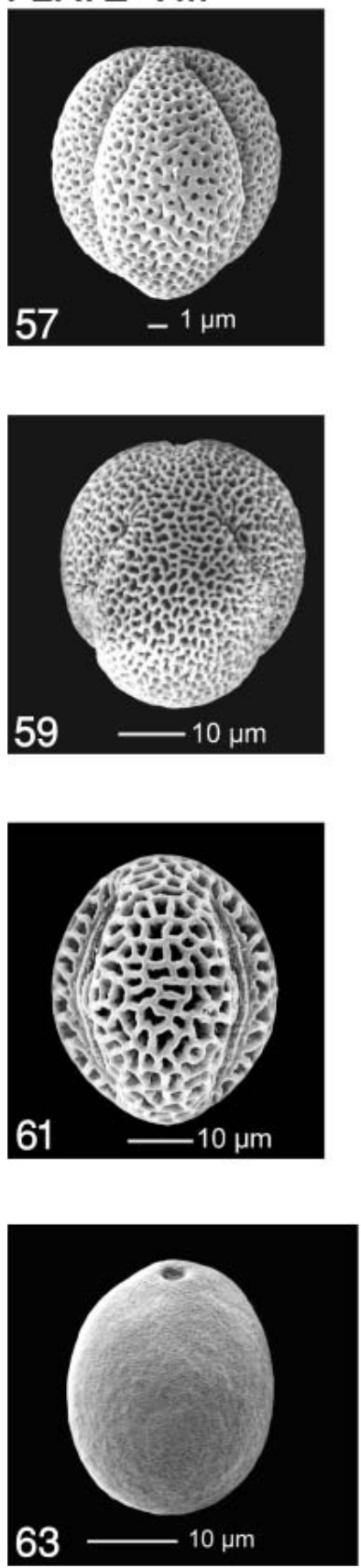
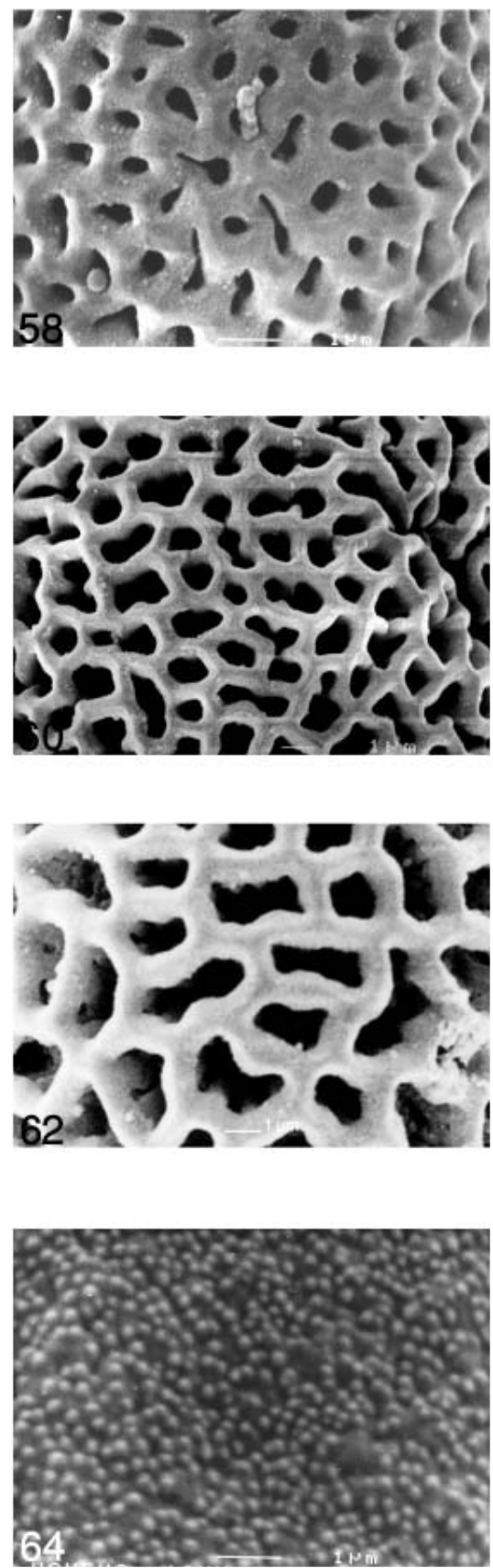


\section{Family Polygonaceae}

Fagopyrum sp.

Plate IX, Figs. 65-66

Shape:Sub prolate.

Size: Polar axis $60 \mu \mathrm{m}$, equatorial axis $30 \mu \mathrm{m}$.

Aperture: Tricolporate.

Exine: $1.2 \mu \mathrm{m}$, sexine is thicker than nexine, reticulate, heterobrochate, muri are smooth and with slopes, lumina are narrow.

\section{Polygonum sp.}

Plate IX, Figs. 67-68

Shape: Spheroidal.

Size: $42 \mu \mathrm{m}$.

Aperture: Pantoporate.

Exine: $4 \mu \mathrm{m}$, sexine much thicker than nexine, reticulate, columella broad, lumina $10 \mu \mathrm{m}$ with numerous rudimentary columellae, width of muri $1 \mu \mathrm{m}$.

\section{Family Proteaceae}

\section{Grevillea sp.}

Plate IX, Figs. 69-70

Shape: Oblate, triangular in polar view

Size: $25 \mu \mathrm{m}$

Aperture: Triporate.

Exine: $1.5 \mu \mathrm{m}$, sexine as much thicker than nexine, nearly reticulate, regulate, Granulate.

\section{Family Rosaceae}

Rosaceae gen. et spec. indet. (1)

Plate IX, Figs. 71-72

Shape: Prolate, circular in polar view.

Size: Polar axis $30 \mu \mathrm{m}$, equatorial axis $20 \mu \mathrm{m}$.

Aperture: Tricolporate.

Exine: $1.5 \mu \mathrm{m}$, sexine slightly thicker than nexine, striate, striations more or less uniformly distributed, partly fused with one another. Colpi broad and granulate. 


\section{PLATE-IX}
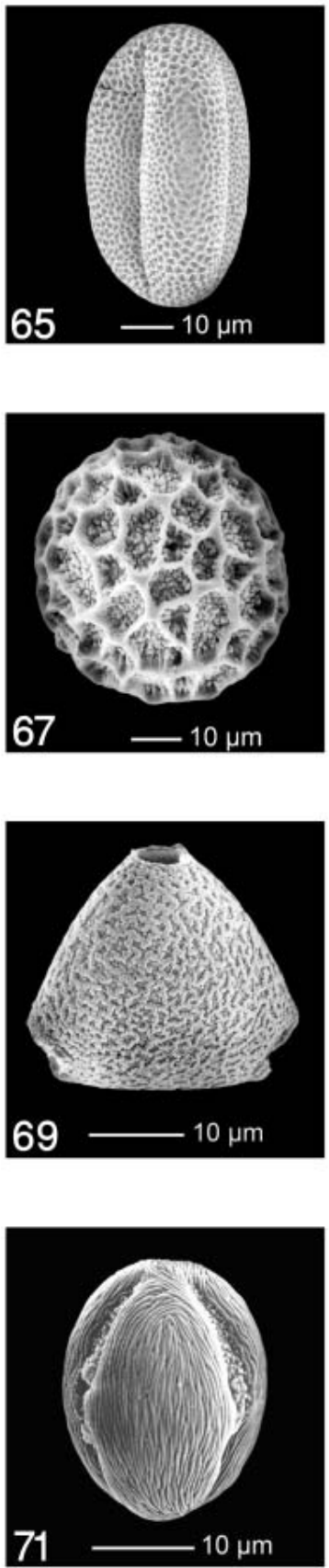
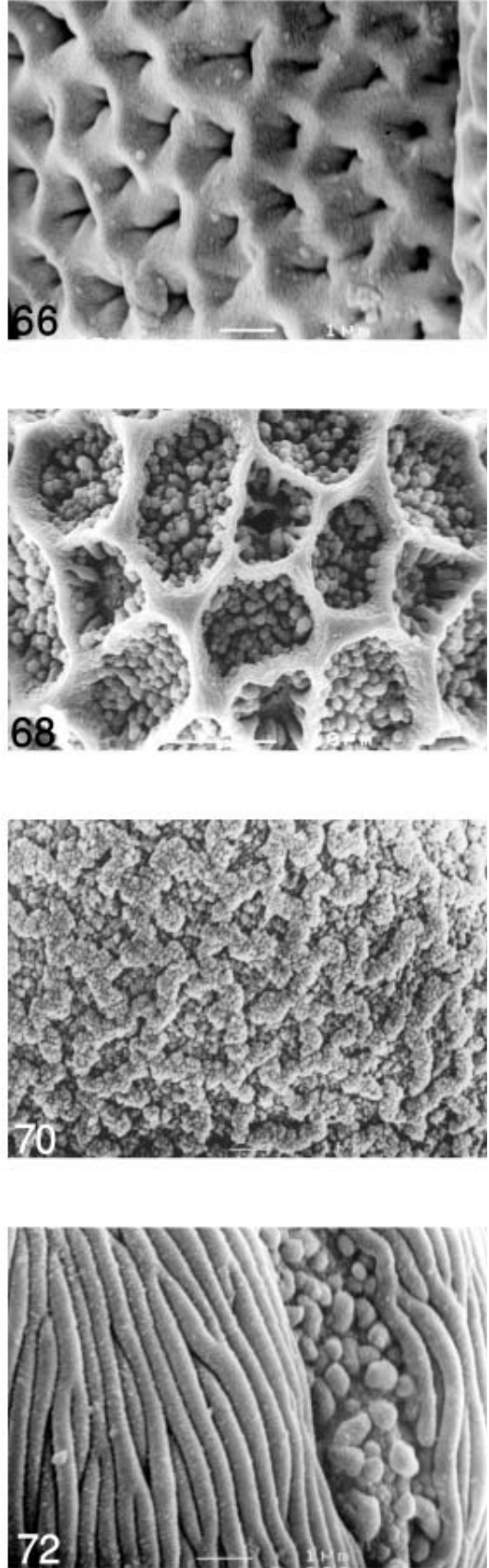
Rosaceae gen. et spec. indet. (2)

Plate X, Figs. 73-74

Shape: Prolate, circular in polar view.

Size: Equatorial axis $22 \mu \mathrm{m}$.

Aperture: Tricolporate.

Exine: $1.5 \mu \mathrm{m}$, sexine slightly thicker than nexine, striations are in the form of sloping rods, randomly oriented, sometimes fused with one another.

Rosaceae gen. et spec. indet. (3)

Plate X, Figs. 75-76

Shape: Prolate, circular in polar view.

Size: Equatorial axis $20 \mu \mathrm{m}$.

Aperture: Tricolporate.

Exine: $1.5 \mu \mathrm{m}$, sexine slightly thicker than nexine, striations are in the form of flat rods, randomly oriented, sometimes fused with one another. Tectum perforated.

Rosaceae gen. et spec. indet. (4)

Plate X, Figs. 77-78

Shape: Prolate, circular in polar view.

Size: Equatorial axis $25 \mu \mathrm{m}$.

Aperture: Tricolporate.

Exine: $1.5 \mu \mathrm{m}$, sexine slightly thicker than nexine, striations are thin and fused, Colporate aperture are broad and distinct.

\section{Family Rutaceae}

\section{Citrus sp.}

Plate X, Figs. $79-80$

Shape: Oblate, circular in polar view.

Size: Equatorial axis 25-30 $\mu \mathrm{m}$.

Aperture: Tetracolporate.

Exine: $2 \mu \mathrm{m}$, reticulated, lumina rounded broad in mesocolpium while small in apocolpium. 

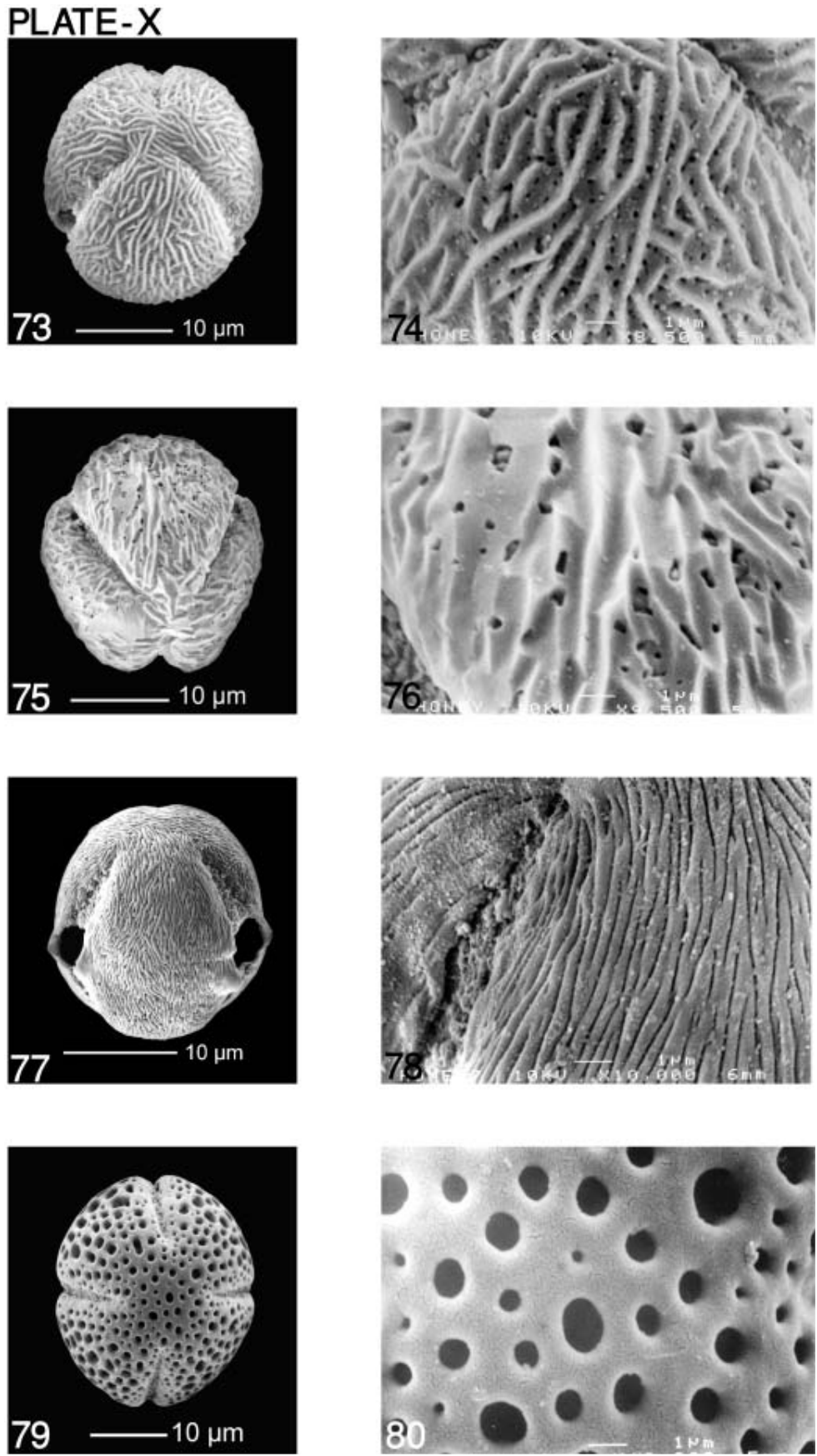


\section{Family Salicaceae}

\section{Salix sp.}

Plate XI, Figs. 81-82

Shape: Prolate, circular in polar view.

Size: Equatorial axis $16 \mu \mathrm{m}$, polar axis $25 \mu \mathrm{m}$

Aperture: Tricolporate, colpi long broad reaching nearly to poles.

Exine: $2 \mu \mathrm{m}$, reticulated, lumina broad in mesocolpium, smaller in apertural area.

\section{Family Sapotaceae}

\section{Aesandra sp.}

Plate XI, Figs. 83-84

Shape: Prolate.

Size: Polar axis $50 \mu \mathrm{m}$, equatorial axis $35 \mu \mathrm{m}$.

Aperture: Tetracolporate, colpi long and gradually broaden towards the poles, end of colpi rounded.

Exine: $1.5 \mu \mathrm{m}$, sexine as thick as nexine, sexine micro-rugulate, perforated and covered by irregularly distributed nano-granules.

\section{Family Urticaceae}

\section{Urtica sp.}

Plate XI, Figs. 85-86

Shape: Oblate.

Size: Equatorial axis 15-20 $\mu \mathrm{m}$.

Aperture: Tricolporate, colpi short and barrow.

Exine: $1.5 \mu \mathrm{m}$, sexine as thick as nexine, sexine micro-echinate, perforated and covered by irregularly distributed nano-granules. Microechinae fused to form clusters.

\section{B. PTERIDOPHYTE}

\section{Pteridaceae gen. et spec. indet.}

Plate XI, Figs. 87-88

Shape: Sub-triangular.

Size: $35 \mu \mathrm{m}$.

Aperture: Trilete.

Exospore: Rugulate in distal pole, rugulae often fused to each other to form irregular depressions (channels) in between them. 

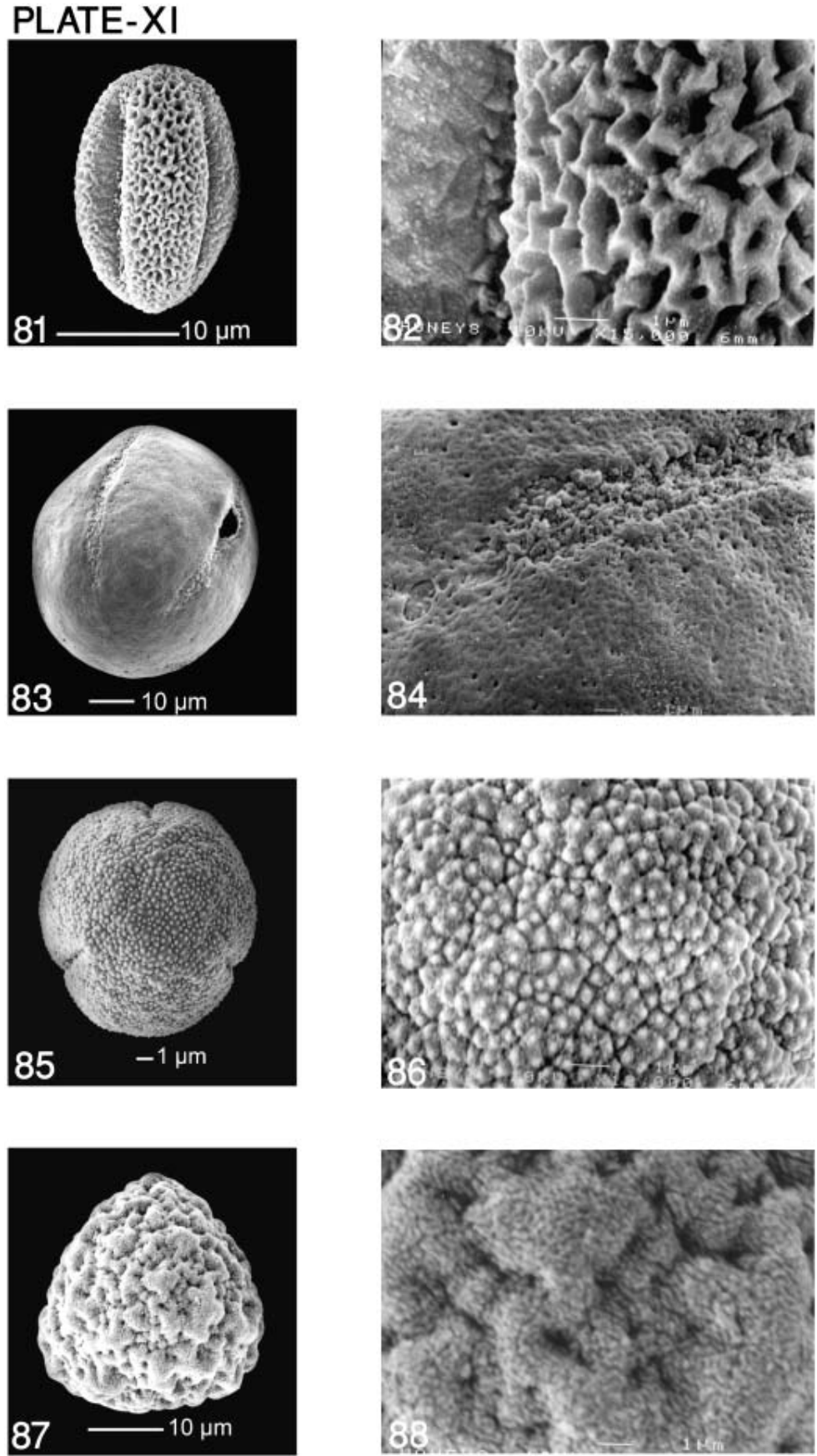


\section{EXPLANATION OF PLATES}

\section{PLATE-I}

Figs. 1-8, Family Acanthaceae

1. Acanthaceae gen. et spec. indet. (1)

2. Details of the tectum of Acanthaceae gen. et spec. indet. (1)

3. Justicia sp.

4. Details of the tectum of Justicia sp.

5. Acanthaceae gen. et spec. indet. (2)

6. Details of the tectum of Acanthaceae gen. et spec. indet. (2)

7. Acanthaceae gen. et spec. indet. (3)

8. Details of the tectum of Acanthaceae gen. et spec. indet. (3)

\section{PLATE-II}

Figs. 9-10, Family Apiaceae; 11-14, Family Araliaceae; 15-16, Family Balsaminaceae

9. Apiaceae gen. et spec. indet.

10. Details of the tectum of Apiaceae gen. et spec. indet.

11. Araliaceae gen. et spec. indet. (1)

12. Details of the tectum of Araliaceae gen. et spec. indet. (1)

13. Araliaceae gen. et spec. indet. (2)

14. Details of the tectum of Araliaceae gen. et spec. indet. (2)

15. Impatiens sp.

16. Details of the tectum of Impatiens sp.

\section{PLATE-III}

Figs. 17-18, Family Betulaceae; 19-20, Family Brassicaceae; 21-22, Family Buxaceae; 23-24, Family Chenopodiaceae

17. Alnus sp.

18. Details of the tectum of Alnus sp.

19. Brassica sp.

20. Details of the tectum of Brassica sp.

21. Sarcococca sp.

22. Details of the tectum of Sarcococca sp.

23. Chenopodiaceae gen. et spec. indet.

24. Details of the tectum of Chenopodiaceae gen. et spec. indet. 


\section{PLATE-IV}

Figs. 25-32, Family Compositae

25. Artemisia sp.

26. Details of the tectum of Artemisia sp.

27. Tubiflorae Compositae gen. et spec. indet. (1)

28. Details of the tectum of Compositae gen. et spec. indet. (1)

29. Tubiflorae Compositae gen. et spec. indet. (2)

30. Details of the tectum of Compositae gen. et spec. indet. (2)

31. Tubiflorae Compositae gen. et spec. indet. (3)

32. Details of the tectum of Compositae gen. et spec. indet. (3)

\section{PLATE-V}

Figs. 33-34, Family Compositae, 35-36, Family Cornaceae, 37-38, Family Corylaceae, 39-40, Family Ericaceae

33. Compositae gen. et spec. indet. (4)

34. Details of the tectum of Compositae gen. et spec. indet. (4)

35. Cornus sp.

36. Details of the tectum of Cornus sp.

37. Corylus sp.

38. Details of the tectum of Corylus sp.

39. Rhododendron $\mathrm{sp}$.

40. Details of the tectum of Rhododendron sp.

\section{PLATE-VI}

Figs. 41-42, Family Fagaceae; 43-44, Family Lamiaceae; 45-46, Family Loranthaceae; 47-48, Family Meliaceae

41. Quercus lanata

42. Details of the tectum of Quercus lanata

43. Lamiaceae gen. et spec. indet.

44. Details of the tectum of Lamiaceae gen. et spec. indet.

45. Loranthaceae gen. et spec. indet.

46. Light Microscope picture of Loranthaceae gen. et spec. indet.

47. Melia azederach

48. Details of the tectum of Melia azederach 


\section{PLATE-VII}

Figs. 49-50, Family Meliaceae; 51-52, Family Myricaceae; 53-54, Family Myrtaceae; 5556 , Family Oleaceae

49. Meliaceae gen. et spec. indet

50. Details of the tectum of Meliaceae gen. at spec. indet.

51. Myrica sp.

52. Details of the tectum of Myrica sp.

53. Syzygium $\mathrm{sp}$.

54. Details of the tectum of Syzygium sp.

55. Fraxinus sp. (1)

56. Details of the tectum of Fraxinus sp. (1)

\section{PLATE-VIII}

Figs. 57-62, Family Oleaceae; 63-64, Family Poaceae

57. Fraxinus sp. (2)

58. Details of the tectum of Fraxinus sp. (2)

59. Jasminum sp.

60. Details of the tectum of Jasminum sp.

61. Ligustrum sp.

62. Details of the tectum of Ligustrum sp.

63. Poaceae gen. et spec. indet.

64. Details of the tectum of Poaceae gen. et spec. indet.

\section{PLATE-IX}

Figs. 65-68, Family Polygonaceae; 69-70, Family Proteaceae; 71-72, Family Rosaceae 65. Fagopyrum sp.

66. Details of the tectum of Fagopyrum sp.

67. Polygonum sp.

68. Details of the tectum of Polygonum sp.

69. Grevillea sp.

70. Details of the tectum of Grevillea sp.

71. Rosaceae gen. et spec. indet. (1)

72. Details of the tectum of Rosaceae gen. et spec. indet. (1) 


\section{PLATE-X}

Figs. 73-78, Family Rosaceae; 79-80, Family Rutaceae

73. Rosaceae gen. et spec. indet. (2)

74. Details of the tectum of Rosaceae gen. et spec. indet. (2)

75. Rosaceae gen. et spec. indet. (3)

76. Details of the tectum of Rosaceae gen. et spec. indet. (3)

77. Rosaceae gen. et spec. indet. (4)

78. Details of the tectum of Rosaceae gen. et spec. indet. (4)

79. Citrus sp.

80. Details of the tectum of Citrus sp.

\section{PLATE-XI}

Figs. 81-82, Family Salicaceae; 83-84, Family Sapotaceae; 85-86, Family Urticaceae; 87-88, Family Pteridaceae

81. Salix sp.

82. Details of the tectum of Salix sp.

83. Aesandra sp.

84. Details of the tectum of Aesandra sp.

85. Urtica sp.

86. Details of the tectum of Urtica sp.

87. Pteridaceae (Pteridophyte spore)

88. Details of the exospore of Pteridaceae spore.

Identification of the pollen up to species level needs a well-identified pollen data bank along with abundant pollen literature of the study area. SEM investigation of honey pollen is not widely recorded in Nepal. Thus, a binomial was only given to the pollen when it could be confirmed by observing a well identified comparative material from herbarium specimens. In other cases only the generic name was used. Some of the pollen could not be identified beyond the family level. A series of pollen articles and manuals (Huang 1972, Nilsson 1973, Huang 1981, Waha 1982, Valdes et al. 1986, Gupta and Sharma 1986, Iwanami et al. 1988, Scotland 1991, Tryon and Lugardon 1991, Moore et al. 1991, Harley 1991, Uffelen 1993, Tissot et al. 1994, Fuhsiung et al. 1995, Jones et al. 1995, Schneider 1996, Unfried 1997, Carine and Scotland 2000, Kapp et al. 2000, Shrestha et al. 2005, Paudayal and Gautam 2011a, 2011b) were found to be very useful for comparison and identification of pollen grains. The taxonomy and the morphological details of palynological assemblages are described in the following paragraphs. The descriptive terminology was followed after Punt et al. (2007). 
In beekeeping, honey bees play a role as micro-manipulators of flowers to produce honey and other hive products. Identification of various bee plants representing potential sources of nectar, pollen, for the honey bees, and their relative importance, is an important pre-requisite for implementing management strategies in particular area to maintain the strength of the colonies and to maximize honey yields. This study dealing with SEM investigation of pollen spectrum of $A$. cerana honeys from Godavari area of Kathmandu Valley provided a unique opportunity to understand the diversity of autochtone bee flora and reliable description regarding the floral types which serve as major or minor nectar and /or pollen sources. The pollens described in this paper are also an expression of its geographical origin of a honey produced by $A$. cerana. The multifloral honey samples contain 44 species of pollen flora belonging to 28 families. The pollen composition was mostly from the plants growing in tropical-subtropical climate zones. Most of the pollen in the palynological assemblages derived from entomophilous plants however the honey sample also contain a significant numbers of anemophilous pollens such as Alnus, Quercus, Corylus, Salix, Chenompodiaceae, Poaceae, etc. Further, this study highlights and signifies the systematic documentation of various nectariferous floras from different parts of the country, thereby helping the beekeepers in the proper management and conservation of autochtone bee colonies.

\section{ACKNOWLEDGEMENTS}

The authors acknowledge the Government of Republic of Austria for providing ÖAD (Österreichische Austauschdienst) fellowships to carry out this study at the Institute of Palaeontology, Vienna University (GEOZENTRUM), Institut für Bienenkunde, Lunz am See and University of Natural Resources and Applied Life Sciences (BOKU), Vienna, Austria. We are grateful to our Ph.D. supervisors Dr. Reinhard Zetter (KNP) and Dr. Hermann Pechhacker (IG) for introducing us in the world of honey pollen.

\section{REFERENCES}

Adams, R. J. and J. L. Morton,1972-1979. An atlas of pollen of the trees and shrubs of eastern Canada and the adjacent United States. Waterloo, ONT: Dept Biol. Univ. Waterloo.

Agwu, C.O.C. and T.O. Akanbi,1985. A palynological study of honey from four vegetation zones in Nigeria. Pollen and Spores, 27:335-348.

Allen, M.Y.,1928. Pollen grains. Bee World, 9:66.

Armbruster, L. and J. Jacobs, 1934-1935. Pollenformen und Honigkerkunfbestimmung. Arch. J. Bienenk., 15(8): 277-308; 16(1, 2B):17-106 (in German).

Armbruster, L. and G. Oenike,1929, Die Pollenformen als Mittel zur Honigherkunftsbestimmung. Bücherei f. Bienenkunde 10. Neumünster I Holst. (in German).

Atwal, A. S. and N.P. Goyal,1974. Apis mellifera turns indifferent to Shain (Plectranthus rugosus). Everyday science, 19:25-26.

Bambara, S. B. and N. A. Leidy,1991. An atlas of selected pollen important to honey bees in the eastern United States. Raleigh, NC: N. Carol. St. Beekeep. Assoc., 38 p.

Betts, A.D.,1923. The identification of pollen sources. Bee World, 5:43-45. 
Betts, A.D.,1925. Notes on pollen identification. Bee World, 7:90.

Bassett, I.J., C.W. Crompton and J.A. Parmelee,1978. An atlas of airborne pollen grains and common fungus spores of Canada.Quebec. Thorne Press Ltd,Can.Dept. Agric. Res.Br.Monogr. 18.

Blackburn, J.S. and J.B. Ford,1993. A study of the foraging behavior of honeybees (Apis mellifera) by the scanning electron microscopic analysis of pollen in honey. Bee Craft, 75:170-175.

Beug, H.-J., 2004. Leitfaden der Pollenbestimmung für Mitteleuropa und angrenzende Gebiete. München, F. Pfeil. (in German).

Carine, M. A. and R.W. Scotland, 2000. 68 taxa and 32 characters: Resolving species relationships using morphological data. In Harley, M.M., C.M. Morton and S. Blackmore (eds) Pollen and spores: Morphology and biology, Royal Botanical Garden, Kew, pp 365-384.

Chanda, S. and P. Ganguly, 1981. Comparative analysis of the pollen content of Indian honeys with reference to entomophily and Anemophily. Proc. IV Intern.,Palynological Conference, Lucknow 4:85-490.

Chaturvedi, M.,1983. Pollen analysis of autumn honeys of Kumaon region. Proc. Ind. Nat. Sci. Acad., 49:125-133.

Chaturvedi, M.,1989. Pollen analysis of some spring honeys from the western Himalayan region. Proc. Ind. Acad. of Sci. (Plant sci.).,99:241-246.

Chen, S.-H. and C. Shen,1990. An ultrastructural study of Formosan honey pollen (I). Taiwania, 35:221-239.

Crane, E.,1981. Endangered honey sources. Bee World, 62(1):3-4.

Crane, E.,1984. Bees, honey and pollen as indicators of metals in the environment. Bee World, 65:47-49.

Deh-Feng, M. and H. Wen Cheng,1981. Apiculture in new China. Bee World, 62:63-166.

Erdtman, G.,1952. Pollen morphology and plant taxonomy angiosperm (An introduction to palynology, vol. 1). Almqvist and Wiksell., Stockholm.

Erdtman, G.,1954. An introduction to pollen analysis. Chronica Botanica Company, Waltham, Mass., USA, $239 \mathrm{p}$.

Erdtman, G.,1960. The acetolysis method: A revised description. Sven. Bot. Tidskr., 54:561-564.

Faegri, K. and J. Iversen,1989. Textbook of pollen analysis. John Wiley and Sons, 328 p.

Fehlmann, C.,1911. Beiträge zur mikroskopischen Untersuchung des Honigs. Mitt. Schweitz. Gesundheitsamtes, 2: 179-221 (in German).

Feller-Demalsy, M.J.,1983. Pollen spectrum of Quebec honeys. Apidologie, 14(3):147-174.

Feller-Demalsy, M.J., J. Parent and A. Strachan,1987. Microscopic analysis of honeys from Alberta, Canada. J. Apicult. Res., 26:123-132.

Ferguson, D.K., R. Zetter and K.N. Paudayal, 2007. The need for the SEM in palaeopalynol- 
ogy, Comptes Rendus Palevol., 6(6-7):423-430.

Focke, E.,1968. The pollen spectrum of Chinese honey. Z. Bienenforsch., 9:195-206.

Fossel, A., 2000. Bienen und Blumen (Herausgeber, Pechhacker, H.). Institut für Bienenkunde, Lunz am See, Austria (in German).

Fuhsiung, W., C. Nanfen, Z. Yulong and Y. Huiqiu,1995. Pollen flora of China. Institute of Botany, Academia Sinica, $461 \mathrm{p}$.

Griebel, C.,1931. Zur Pollenanalyse des Honigs. Z. f. Unters. der Lebensmittel, 61(3):241-306 (in German).

Gupta, H.P. and C. Sharma,1986. Pollen flora of North-West Himalaya. Indian association of Palynostratigraphers, Janki Bhawan, Narhi, Lucknow, India, 181 p.

Harley, M. M.,1991. The pollen morphology of the Sapotaceae. Kew bulletin, 46(39):379-491.

Herrera, F. and L.E. Urrego,1996. Atlas de polen de plantas u' tiles y cultivadas de la Amazonia colombiana. Santafe' de Bogota': Tropenbos Colombia and Fund. Erigaie, Estud, Amazonia Colomb 11 (in Spanish).

Huang, Tseng-Ching, 1972. Pollen flora of Taiwan. National Taiwan University, Botany Department press, $297 \mathrm{p}$.

Huang, Tseng-Ching,1981. Spore flora of Taiwan. National Taiwan University, Botany Department press, $112 \mathrm{p}$.

ICIMOD,1996. Promotion and development of beekeeping through preservation of indigenous Apis cerana. Annual Project Progress Report Submitted to the Federal Chancellory of Austria.

Iwanami, Y., T. Sasakuma and H. Yamada,1988. Pollen: Illustrations and scanning electronmicrographs. Springer-Verlag, $198 \mathrm{p}$.

Jato, M.V., A. Sala-Llinares, M. I. Iglesias and M. Suarez-Cervera,1991. Pollen of honeys from north-western Spain. J. Apicult. Res., 30:69-73.

Jato, M.V., M.I. Iglesias and V. Rodriguez-Gracia,1994. A contribution to the environmental relationship of the pollen spectra of honeys from Ourense (NW Spain). Grana, 33:260-267.

Jones, G.D., V.M. Bryant jr., M.H. Lieux, S. D. Jones and P.D. Lingren,1995. Pollen of the southern United States: With emphasis on melissopalynology and entomopalynology. AASP contribution series number 30 , The American Association of Stratigraphic Palynologists Foundation, Dallas, Texas, $104 \mathrm{p}$.

Jones, G.D. and J.R. Coppedge,1998. Pollen analyses of the boll weevil exoskeleton. In Bryant, V. M.Jr. and J.H. Wrenn (eds) New developments in palynomorph sampling, extraction, and analysis. American Association of Stratigraphic Palynologists Foundation, Dallas, Texas. pp 121-125.

Joshi, S.R.,1999. Physico-chemical and melissopalynological characteristics of Nepalese honey. Ph.D. Thesis, University of Agricultural Sciences, Vienna, Austria.

Kafle, G.P.,1984. A general survey of bee flora in and around Kathmandu Valley. Nepalese 
Journal of Agriculture, 15:89-99.

Kafle, G.P.,1992. Salient features of beekeeping in Nepal. In Verma, L.R. (ed) Honeybees in mountain agriculture. New Delhi, Oxford and IBH Publishing Co. Pvt. Ltd., pp 155-162.

Kapp, O.R., O.K. Davis and J.E. King, 2000. Ronald O. Kapp's pollen and spores. The American Association of Stratigraphic Palynologists Foundation, $279 \mathrm{p}$.

Köppler, K., G. Vorwohl and N. Koeniger, 2007. Comparison of pollen spectra collected by four different subspecies of the honey bee Apis mellifera. Apidologie, 38:341-353.

Loublier, Y., M.L.Piana, M.H. Pham-Delegue and R. Borneck,1994. Characterization of monofloral lavender honeys. Grana, 33:231-238.

Lobreau-Callen, D. and F. Damblon,1994. Pollen spectra of honey Apis mellifera L. and vegetation zones in west tropical Mediterranean Africa. Grana, 33:245-258.

Louveaux, J., A.Maurizio and G.Vorwohl,1978. Methods of Melissopalynology. Bee World, 59:139-153.

Maskey, M.,1989. Inter-relationship between bees and flowers in Kathmandu Valley. Paper presented at the Fourth International Conference on Apiculture in Tropical Climates, Cairo, Egypt. Maskey, M.,1992. Mountain women and beekeeping in Nepal. In Verma, L.R. (ed) Honey bees in mountain agriculture. New Delhi, Oxford and IBH Publishing Co. Pvt. Ltd., pp 119-132.

Maurizio, A.,1951, Pollen analysis of honey. Bee World, 32:1-5.

Moar, N.T.,1993. Pollen grains of New Zealand dicotyledonous plants. Lincoln, Canterb., N.Z., Manaaki Whenua Press.

Molan, P.C.,1998. The limitations of the methods of identifying the floral source of honeys. Bee World, 79:59-68.

Moore, P.D., J.A. Webb and M.E. Collinson,1991. Pollen analysis. London, Blackwell Sci. Publ., $216 \mathrm{p}$ (2nd edition).

Nair, P. K. K.,1964. A pollen analytical study of Indian honeys. J. Ind. Bot. Soc., 43:179-191.

Nilsson, S.,1973. Menyanthaceae Dum. (taxonomy by Robert Ornduff). World pollen and spore flora 2, the Almqvist and Wiksell Periodical Company, Stockholm, pp 1-19.

Nilsson, S., J. Praglowski and L. Nilsson,1977. Atlas of airborne pollen grains and spores in northern Europe. Natur and Kultur, Sweden.

Ogden, E.C., G.S. Raynor, J.V. Hayes, D.M. Lewis and J. H. Haines, 1974. Manual for sampling airborne pollen. New York, Hafner Press.

Partap, U. and L.R. Verma,1996. Impact of food resource availability on growth and development of Apis cerana colonies in the Kathmandu Valley of Nepal. Ecoprint, 3:21-29.

Partap, U.,1997. Bee flora of the Hindu Kush-Himalayas: Inventory and management. International Centre for Integrated Mountain Development, Kathmandu Nepal.

Paudayal, K.N. and I. Gautam, 2011a. Scanning electron microscopic studies on surface 
pattern of the pollen loads from Apis cerana in Jajarkot district. Nepal Journal of Science and Technology, 12:340-349.

Paudayal, K.N. and I. Gautam, 2011b. Palynological study of pollen loads of Apis cerana in Bajhang district, west Nepal using scanning electron microscope. Perspectives on Higher Education, Journal of TUTA University Campus, 6:77-86.

Punt, W., P.P. Hoen, S. Blackmore, S. Nilsson and A.L. Thomas, 2007. Glossary of pollen and spore terminology. Review of Palaeobotany and Palynology, 143:1-81.

Qiao, B.-S., 2004. Color atlas of air-borne pollen and plants in China. Beijing, Peking Union Med. Coll. Press.

Ridgeway, J.E. and J.J. Skvarla,1969. Scanning electron microscopy as an aid to pollen taxonomy. Ann. Mo. Bot. Gard., 56:121-124.

Rowley, J.R., J.J. Skvarla and E.L.Vezey,1988. Evaluating the relative contributions of SEM, TEM, and LM to the description of pollen grains. J. Palynol. (India), 23-24:27-28.

Saraf, S.K.,1972. Bee flora of Kashmir. Indian Bee Journal, 34:1-10.

Schneider, W.,1996. Zur Pollenmorphologie ausgewählter rezenter und fossiler Vertreter der Oleaceae. Diplomarbeit zur Erlangung des akademischen grades Magistra der Naturwissenschaften an der Formal- und Naturwissenschaftlichen Fakultät der Uni Wien (M. Sc. thesis) (in German).

Scotland, R.W.,1991. A systematic analysis of pollen morphology of Acanthaceae genera with contorted corollas. In Blackmore S. and S. H. Barnes (eds) Pollen and spores, pattern and diversification. Systematics Association, pp 269-289.

Sen, J. and D.Banerjee,1956. A pollen analysis of Indian honey. Bee World, 37(3):52-54.

Sharma, M. and P.K.K. Nair,1965. Pollen analysis of some honeys from Uttar Pradesh. Ind. J. Hor., 22:46-51.

Shrestha, K.K., K.N. Paudayal and D.K. Ferguson, 2005. Pollen morphology of the Himalayan Viola (Violaceae). Nepal Journal of Science and Technology, 5:73-80.

Singh, M.P.,1989. Melissopalynology and identification of Himalayan races of honeybees by computer assisted multivariate analysis. Ph.D. Thesis, Himachal Pradesh University, Shimla, India.

Singh, Y.,1983. Beekeeping in Uttar Pradesh: A review. Indian Bee Journal, 45:84-92.

Skvarla, J.J. and D.A. Larson,1965. An electron microscopic study of pollen morphology in the Compositae with special reference to the Ambrosiinae. Grana, 6:210-269.

Suryanarayana, M.C. and C.V. Thakar,1966. Studies on ornamental garden plants as bee forage I Phlox drummondii, Hk. F. Ind. Bee J., 28:17-20.

Sowunmi, M.A.,1976. The potential value of honey in palaeopalynology and archaeology. Rev. Palaeobot. Palynol., 21:171-185.

Suryanarayana, M.C.,T.S.Seethalakshmi and R.P. Phadke,1977. Microscopical analysis of 
Indian honeys 1. Honeys from Litchi (Nephelium litchi) and Jamun (Syzygium cumini). Abstract, IV International Palynological Conference.

Telleria, M.C.,1988. Palynological analysis of honeys from the north-west of the province of Buenos Aires. Apidologie, 19(3):275-290.

Terrab, A., M.J. Diez and F.J. Heredia, 2003. Palynological, physicochemical and colour characterisation of Moroccan honeys. I. River Red Gum (Eucalyptus camaldulensis Dehnl.) honey. International Journal of Food Science and Technology, 38:379-386.

Tissot, C., H. Chikhi and T.S. Nayar,1994. Pollen of wet evergreen forests of the western ghats of India. Publications du department d'ecologie, Institut Francais de Pondichery, $133 \mathrm{p}$.

Todd, F.E. and Vansell, G.H.,1942. Pollen grains in nectar and honey. The Journal of Economic Entomology, 35:728-731.

Tryon, A.F. and B.Lugardon,1990. Spores of the pteridophyta: Surface, wall structure and diversity based on electron microscope studies. Springer-Verlag, $648 \mathrm{p}$.

Uffelen, G.A. van,1993. Morphogenesis and evolution of the spore wall of Polypodiaceae (Filicales). Rijksherbarium/Hortus Botanicus, Leiden, $180 \mathrm{p}$.

Unfried ,S., 1997. Zur Pollenmorphologie rezenter und fossiler Vertreter der Gattung Rhododendron. Diplomarbeit zur Erlangung des akademischen grades Magistra der Naturwissenschaften an der Formal- und Naturwissenschaftlichen Fakultät der Uni Wien (M. Sc. Thesis) (in German).

Valdes, B., M.J. Diez and I. Fernandez, 1986. Atlas polinico de andalucia occidental. Instituto de Desarrollo Regional $N^{\circ} 43$, Universidad de Sevilla, Excma. Diputacion de Cadiz, $451 \mathrm{p}$ (in Spanish).

Valencia-Barrera, R.M., M. A. Fombella-Bianco, D. Fernandez-Gonzalez and T. E. Diaz,1994. Pollen spectra of honey from different phytogeographical regions of the Leon province, NW Spain. Grana, 33:268-275.

Van Laere, O., A. Lagasse and M., de. Mets,1969. Use of the scanning electron microscope for investigating pollen grains isolated from honey samples. J. Apicult. Res., 8:139-145.

Varis, A.L., J. Helenius and K. Koivulehto,1982. Pollen spectrums of Finnish honey. J. Sci. Agric. Soc. Finl., 54(5):403-413.

Vezey, E.L. and J.J. Skvarla,1990. Computerized feature analysis of exine sculpture patterns. Rev. Palaeobot. Palynol., 64:187-196.

Vezey, E.L., J.J. Skvarla and S.S.Vanerpool,1991. Characterizing pollen sculpture of three closely-related Capparaceae species using quantitative image analysis of scanning electron micrographs. In Blackmore, S. and S. J. Barnes (eds) Pollen and spores: Patterns of diversification. Oxford, Clarendon Press, Syst. Assoc. Sp. Vol. 44, pp 291-300.

Vishnu-Mittre,1958. Pollen content of some Indian honeys. J. Sci. and Industr. Res., 17:123-124.

Waha, M.,1982. Zur Ultrastruktur und Funktion pollenverbindender Fäden bei Angiospermen, insbesondere der Vscinfäden bei Ericaceae. Dissertation zur Erlangung des Doktorgrades an 
der Formal- und Naturwissenschaftlichen Fakultät der Uni Wien (PhD Thesis) (in German).

Wang, H., L.-M. Gao, X.-L. Zhang, L.-H. Zhou and Y.-X. Feng (ed Wei, Z.-X.), 2003. Pollen flora of seed plants. Kunming, Yunnan Sci. and Technol. Press.

Wodehouse, R.P.,1935. Pollen grains. McGraw-Hill, New York, 574 p.

Zander, E. and A. Maurizio,1975. Der Honig. Handbuch der Bienenkunde 6, Ulmer, Stuttgart, $212 \mathrm{p}$ (in German).

Zander, E.,1935,1937, 1941,1949,1951. Beiträge zur Herkunftsbestimmung bei Honig. I-IV. I: Reichsfachgruppe Imker. Berlin; II, III, V: Liedloff, Loth and Michaelis, Leipzig; IV; Ehrenwirth, München (in German).

Zetter, R.,1989. Methodik und bedeutung einer routinemäßig kombinierten lichtmikros- kopischen und rasterelektronenmikroskopischen untersuchung fossiler mikrofloren. Cour. Forsch.Inst. Senckenberg, Frankfurt am Main. 109:41-50 (in German).

\section{AUTHOR'S ADDRESS}

Khum Narayan Paudayal ${ }^{1}$

Central Department of Geology, Tribhuvan University, Kirtipur, Kathmandu (email: khum99@gmail.com)

Ishan Gautam ${ }^{2}$

Natural History Museum, Tribhuvan University, Swayambhu, Kathmandu 